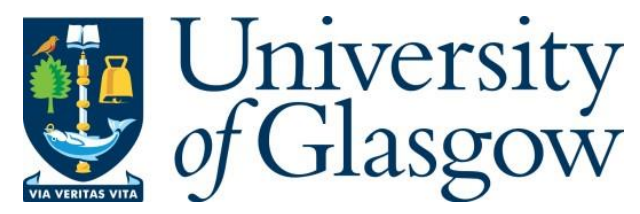

Reinsberg, B. and Westerwinter, O. (2019) The global governance of international development: documenting the rise of multi-stakeholder partnerships and identifying underlying theoretical explanations. Review of International Organizations, (doi: 10.1007/s11558-019-09362-0).

There may be differences between this version and the published version. You are advised to consult the publisher's version if you wish to cite from it.

http://eprints.gla.ac.uk/188236/

Deposited on: 14 June 2019

Enlighten - Research publications by members of the University of Glasgow http://eprints.gla.ac.uk 


\title{
Structural shape optimization of three dimensional acoustic problems with isogeometric boundary element methods
}

\author{
L.L. Chen ${ }^{\mathrm{a}}$, H. Lian ${ }^{\mathrm{b}}$, Z. Liu ${ }^{\mathrm{c}}$, H.B. Chen ${ }^{\mathrm{d}}$, E. Atroshchenko ${ }^{\mathrm{e}}$, S.P.A. Bordas ${ }^{\mathrm{b}, \mathrm{f}, \mathrm{g}, *}$ \\ ${ }^{a}$ College of Architecture and Civil Engineering, Xinyang Normal University, PR China \\ ${ }^{b}$ Institute for Computational Engineering, Faculty of Science, Technology and Communication, University \\ of Luxembourg, Luxembourg \\ ${ }^{c}$ College of Science and Engineering, University of Glasgow, UK \\ ${ }^{d}$ CAS Key Laboratory of Mechanical Behavior and Design of Materials, Department of Modern Mechanics, \\ University of Science and Technology of China, Hefei 230026, PR China \\ ${ }^{e}$ School of Civil and Environmental Engineering, University of New South Wales, Sydney, Australia \\ ${ }^{f}$ School of Engineering, Cardiff University, The Parade, CF24 3AA, Cardiff, UK \\ ${ }^{g}$ China Medical University Hospital, China Medical University, Taichung, Taiwan, R.O.C.
}

\begin{abstract}
The boundary element method (BEM) is a powerful tool in computational acoustics, because the analysis is conducted only on structural surfaces, compared to the finite element method (FEM) which resorts to special techniques to truncate infinite domains. The isogeometric boundary element method (IGABEM) is a recent progress in the category of boundary element approaches, which is inspired by the concept of isogeometric analysis (IGA) and employs the spline functions of CAD as basis functions to discretize unknown physical fields. As a boundary representation approach, IGABEM is naturally compatible with CAD and thus can directly perform numerical analysis on CAD models, avoiding the cumbersome meshing procedure in conventional FEM/BEM and eliminating the difficulty of volume parameterization in isogeometric finite element methods. The advantage of tight integration of CAD and numerical analysis in IGABEM renders it particularly attractive in the application of structural shape optimization because (1) the geometry and the analysis can be interacted, (2) remeshing with shape morphing can be avoided, and (3) an optimized solution returns a CAD
\end{abstract}

\footnotetext{
* Corresponding author

Email address: stephane.bordas@uni.lu (S.P.A. Bordas)
} 
geometry directly without postprocessing steps. In the present paper, we apply the IGABEM to structural shape optimization of three dimensional exterior acoustic problems, fully exploiting the strength of IGABEM in addressing infinite domain problems and integrating $\mathrm{CAD}$ and numerical analysis. We employ the Burton-Miller formulation to overcome fictitious frequency problems, in which hyper-singular integrals are evaluated explicitly. The gradient-based optimizer is adopted and shape sensitivity analysis is conducted with implicit differentiation methods. The design variables are set to be the positions of control points which directly determine the shape of structures. Finally, numerical examples are provided to verify the algorithm.

Keywords: acoustics; exterior domain; isogeometric analysis; boundary element method; shape sensitivity analysis; shape optimization

\section{Introduction}

Acoustics found applications in a wide range of engineering areas, such as noise control, sonar for underwater navigation, non-destructive testing, ultrasound medical imaging, seismology, bioacoustics, electroacoustic communications, etc. The finite element method (FEM) and the boundary element method (BEM) are two commonly used techniques in simulating acoustics problems. Although the FEM is the dominant numerical method in a number of engineering applications, the BEM has some appealing features by reducing the dimension by one: only the boundary of the domain needs to be discretized and the Sommerfeld radiation condition at infinity can be fulfilled automatically. As such, BEM not only reduces the number of degree of freedoms considerably, but more importantly, facilitates the geometric modeling and alleviates meshing burden [10]. The matrices in BEM are non-symmetric and densely populated, which restrains its application to large scale problems. In order to improve efficiency, various acceleration techniques were developed, such as fast multiple methods (FMM) [24], the wideband FMM [65], the fast wavelet transforms [9], the precorrected fast 
Fourier transformation [48], and the adaptive cross approximation method [4]. In addition, with the partition of unity method, the BEM can be enriched to reduce the number of elements per wave length $[47,8]$, whose errors at element ends can be further reduced by using trigonometric shape functions [43]. In order to exploit the benefits of both FEM and BEM, the coupled scheme of BEM/FEM was proposed for structuralacoustic analysis in $[22,64]$ and for corresponding sensitivity analyses $[14,11,13]$.

Finding the optimal shape of a structure or a component subject to certain constraints is central to engineering design. Although shape optimization methods based on numerical analysis and mathematical programming are regarded as a pace-setting technology to rationalize and automate design processes, their application in industry is still limited. This problem emanates from the disconnection between the geometric models used in Computer-Aided Design (CAD) and that used in numerical analysis. The geometric models in CAD are boundary-represented and constructed with splines such as Non-Uniform Rational B-splines (NURBS), whereas the numerical analysis algorithms such as the FEM rely on polyhedral meshes over the interior domain of the structure [38]. Hence, a cumbersome meshing procedure is imperative in the preprocessing step, which is significantly complicated and often involves intensive human intervention in spite of significant recent advances where millions of tetrahedra are generated within one second only [37]. Such geometry-to-meshing mapping problem is even more severe in shape optimization, because (1) the geometry varies at each step of the iterative procedure and thus requires repetitive meshing procedures, and (2) the data of the geometry and the physical field need to be communicated between FEM meshes and NURBS. An approach which can alleviate this meshing burden to some extent is to deform the mesh once it is constructed in the preprocessing step, but this leads to a large number of design variables and requires smoothing to eliminate the oscillatory boundaries ([16], [15], [32], [66]). Alternatively, implicit representations of the geometry, for example level set methods [61], can be employed to track the moving 
boundary within a fixed grid, but the method is susceptible to numerical diffusion.

Hughes et al. [26] proposed isogeometric analysis (IGA) to bridge the CAD and numerical analysis. The key idea of IGA is to use the spline functions in CAD models as basis functions to discretize the unknown fields of partial differential equations. An immediate advantage of IGA is that CAD models can be directly analyzed without recourse to meshing. In addition, IGA possesses other advantages such as geometric exactness, flexible order elevation and $k$-refinement, high order continuous fields, etc. The application of IGA has been extended to numerous areas, such as structural vibration [18], shell analysis [7], fluid problems [3], fluid-structure interaction [2], contact [19], composite delamination [41], acoustics [27], etc. In parallel, more advanced CAD model techniques are incorporated into IGA, including T-splines [1], PHT-splines [42], and subdivision surfaces [17], which make local refinement possible. Apart from that, the Bézier extraction technique is put forward for the ease of implementation of IGA in existing FEM codes [51]. A review of the implementation aspects of IGA can be seen in [40].

The IGA was initially developed in the context of finite element methods (IGAFEM) [40]. An inherent difficulty of IGAFEM is that a volumetric parameterization is needed, which is far from a trivial task in practice because CAD models are boundary represented. Although some progress have been made along this line [62, 63], much difficulties, akin to those encountered in hexahedron mesh generation, remain. To achieve a seamless integration between $\mathrm{CAD}$ and numerical analysis, isogeometric boundary element methods (IGABEM) were proposed: the spline basis functions of CAD models are used to discretize the Boundary Integral Equations. As a boundaryrepresentation approach, IGABEM is naturally compatible with CAD. IGABEM was first applied to potential flow [49] and elastostatic analysis [54, 53, 52]. The method exhibits superior accuracy per degree of freedom and is naturally able to simulate complex geometries. The application of IGABEM in fracture mechanics $[46,45]$ further 
demonstrated its potential in dealing with moving boundaries and singularities. So far, IGABEM has been applied successfully in wave-resistance [23], elasto-plastic inclusions [6, 5], structural-acoustic coupling [36], electromagnetics [56], etc. Recent continuations include the fast multipole method [55, 31], proper generalized decomposition [31], Galerkin scheme [39], and adaptive refinement based on a posterior error estimation $[21,20]$.

Due to the merits of both IGA and conventional BEM, IGABEM possesses great advantages in acoustics and shape optimization. Simpson et al. [57] were the first to apply IGABEM for acoustic analysis, where both interior and exterior problems in three dimension were considered. [44] enriched IGABEM to capture shorter wave lengths with fewer degrees of freedoms. Special integration routines for hypersingular and nearly singular integrals that arise in IGABEM were presented in [58].

Shape optimization of IGABEM is initially applied in linear elasticity [29, 33, 34, 59] and hydrodynamics [28]. Chen et al. [12,35] applied IGABEM to the sensitivity analysis and shape optimization for acoustic problems in 2D, which fully exploits the capability of IGABEM to address infinite domain problems and integrating CAD and numerical analysis.

The present paper is devoted to extending previous work [12] to three dimensional acoustic shape optimization. The remainder of the paper is organized as follows. Section 2 reviews the fundamental formulations of IGABEM for acoustic analysis, where NURBS and the evaluation of singular integrals are detailed since they play a key role in the implementation of IGABEM. Section 3 introduces the sensitivity analysis and gradient-based optimization algorithms used in the paper. The fourth section provides numerical examples and is followed by conclusions. 


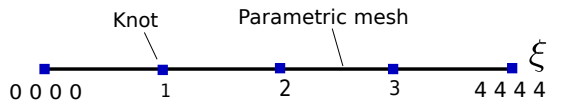

Fig. 1: Knot vector $\{0,0,0,0,1,2,3,4,4,4,4\}$.

\section{Non-Uniform Rational B-Splines (NURBS)}

For completeness, this section briefly reviewed some basic concepts in NURBS, which is fundamental to the isogeometric analysis. The readers are referred to [26] for more details. An important concept in NURBS is knot vector, which is a set of non-decreasing real numbers, written as follows

$$
\Xi=\left[\xi_{1}, \xi_{2}, \cdots, \xi_{n+p+1}\right], \quad \xi_{a} \in \mathbb{R}
$$

where $a$ is the knot index, $p$ is the polynomial order, and $n$ is the number of the basis functions. Knot vector can be regarded as a one dimensional parametric space. See Fig. 1.

Given a knot vector, the B-spline basis functions are written as Coxde Boor recursion formula (Cox, 1971; de Boor, 1972)

$$
N_{a, 0}(\xi)= \begin{cases}1 & \text { if } \quad \xi_{a} \leq \xi<\xi_{a+1} \\ 0 & \text { otherwise }\end{cases}
$$

for $p=0$, and

$$
N_{a, p}(\xi)=\frac{\xi-\xi_{a}}{\xi_{a+p}-\xi_{a}} N_{a, p-1}(\xi)+\frac{\xi_{a+p+1}-\xi}{\xi_{a+p+1}-\xi_{a+1}} N_{a+1, p-1}(\xi),
$$

for $p \geq 1$.

B-spline basis functions have several favorable properties such as local support, point wise non-negativity, linear independence, which are amenable to numerical analysis (Fig. 2). 


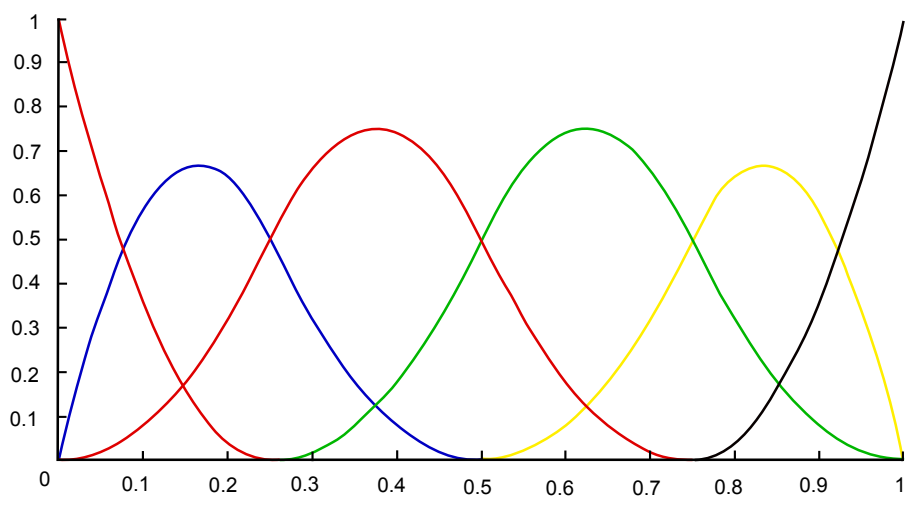

Fig. 2: NURBS basis functions

The B-spline curve can be constructed as a linear combination of B-spline basis functions with control points:

$$
\mathbf{x}(\xi)=\sum_{i=1}^{n} N_{a, p}(\xi) \mathbf{P}_{a, p},
$$

where the coefficients $\mathbf{P}_{a, b}$ are the coordinates of control points. Hence, a B-spline curve is in essence is a mapping from a one dimensional parametric space to physical space. For two dimensional parametric spaces with the following knot vectors in each dimension:

$$
\begin{array}{cc}
{\left[\xi_{1}, \xi_{2}, \cdots, \xi_{n+p+1}\right],} & \xi_{a} \in \mathbb{R} \\
{\left[\eta_{1}, \eta_{2}, \cdots, \eta_{m+l+1}\right],} & \xi_{b} \in \mathbb{R}
\end{array}
$$

the B-spline surface can be constructed with the tensor product property,

$$
\mathbf{x}(\xi, \eta)=\sum_{a=1}^{n} \sum_{b=1}^{m} N_{a, p}(\xi) N_{b, l}(\eta) \mathbf{P}_{a, b}
$$

where $n$ and $m$ are number of basis function in each dimension, respectively. It is noted that due to lack of Kronecker delta property (Fig. 2), the control points of B-splines are not necessarily located on the surface, as shown in Fig 3. 


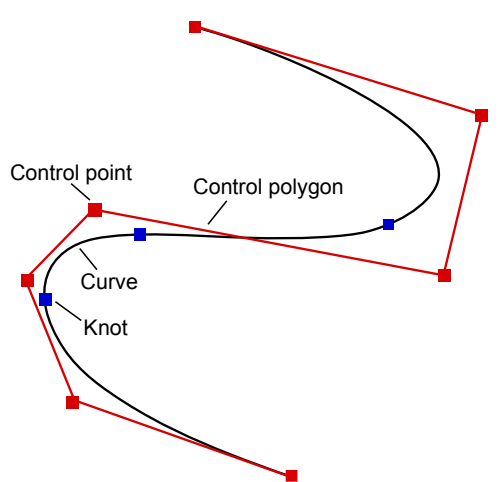

(a)

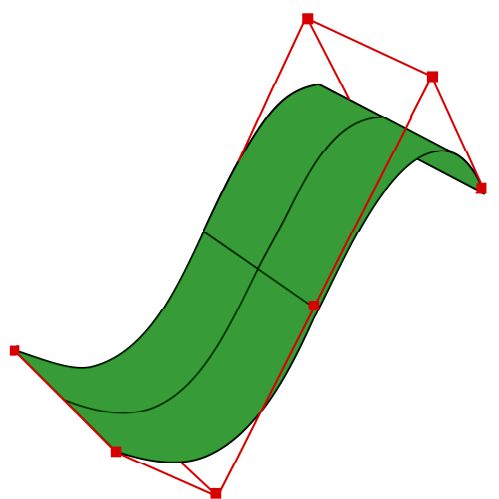

(b)

Fig. 3: NURBS geometries. (a) NURBS curve; (b) NURBS surface

NURBS are extension of B-splines by associating a weight with each control point. Using NURBS, designers can obtain more control of the represented curve without increasing the number of control points or increasing the degree, and can also represent exactly some curves with conic sections, such as circles and ellipses. The NURBS basis functions are constructed from B-spline basis functions, which is in two dimensions written as

$$
R_{a, b}(\xi, \eta)=\frac{N_{b, p}(\xi) N_{b, l}(\eta) w_{a, b}}{W(\xi, \eta)}
$$

where $w$ is referred to as the weight, and

$$
W(\xi, \eta)=\sum_{a=1}^{n} \sum_{b=1}^{m} N_{a, p}(\xi) N_{b, l}(\eta) w_{a, b}
$$

The NURBS surfaces are defined using NURBS basis functions and control points in a similar manner as B-spline surfaces,

$$
\mathbf{x}(\xi, \eta)=\sum_{a=1}^{n} \sum_{b=1}^{m} R_{a, p}(\xi) R_{b, l}(\eta) \mathbf{P}_{a, b}
$$


For succinctness, in the following we drop the notation $p$ and use the global index $A$ to iterate over basis functions or control points, then the above equation can be rewritten as

$$
\mathbf{x}(\xi, \eta)=\sum_{A=1}^{N_{A}} R_{A}(\xi, \eta) \mathbf{P}_{A}
$$

By using knot insertion operator, more control points can be introduced without changing the structural shape. This property is beneficial to improvement of the accuracy on approximating the physical fields while retaining the geometric accuracy using $h$-refinement scheme.

\section{IGABEM for acoustic state analysis}

\subsection{IGABEM formulation with Burton-Miller method}

If $p$ denotes the sound pressure, $\Omega$ the fluid domain, $k=\omega / c$ the wave number ( $\omega$ is the angular frequency and $c$ is the wave speed in the acoustic medium $\Omega$ ), the Helmholtz equation for acoustic problems is expressed as

$$
\forall \mathbf{x} \in \Omega, \quad \nabla^{2} p(\mathbf{x})+k^{2} p(\mathbf{x})=0,
$$

where $\mathbf{x}$ is the field point located in the domain. The conventional boundary integral equation (CBIE) for the Helmholtz equations suitable for acoustic scattering is

$$
c(\mathbf{s}) p(\mathbf{s})=\int_{S} G(\mathbf{s}, \mathbf{x}) q(\mathbf{x}) \mathrm{d} S(\mathbf{x})-\int_{S} F(\mathbf{s}, \mathbf{x}) p(\mathbf{x}) \mathrm{d} S(\mathbf{x})+p_{\text {inc }}(\mathbf{s}),
$$

where $S$ is the structural surface, $\mathbf{x}$ and $\mathbf{s}$ are the field point and source point located on the boundary, respectively, $c(\mathbf{s})$ is the jump term which is equal to $\frac{1}{2}$ for smooth boundaries, and $q(\mathbf{x})$ stands for the flux, which is the normal derivatives of pressure $p(\mathbf{x})$, i.e. $q(\mathbf{x})=\frac{\partial p(\mathbf{x})}{\partial \mathbf{n}(\mathbf{x})}$. The term $p_{\text {inc }}(\mathbf{s})$ indicates the incident wave acoustic pressure 
which can be ignored in acoustic radiation problems. $G(\mathbf{s}, \mathbf{x})$ is the Green function which is written as

$$
G(\mathbf{s}, \mathbf{x})=e^{\mathrm{i} k r} / 4 \pi r
$$

with $r=|\mathbf{s}-\mathbf{x}| . F(\mathbf{s}, \mathbf{x})$ is the normal derivatives of $G(\mathbf{s}, \mathbf{x})$ :

$$
F(\mathbf{s}, \mathbf{x})=\frac{\partial G(\mathbf{s}, \mathbf{x})}{\partial \mathbf{n}(\mathbf{x})}=-\frac{e^{\mathrm{i} k r}}{4 \pi r^{2}}(1-\mathrm{i} k r) \frac{\partial r}{\partial \mathbf{n}(\mathbf{x})}
$$

By differentiating Eq. (13) with respect to the normal at the source point $\mathbf{n}(\mathbf{s})$, we can obtain the hypersingular boundary integral equations (HBIE) as

$$
c(\mathbf{s}) q(\mathbf{s})=\int_{S} K(\mathbf{s}, \mathbf{x}) q(\mathbf{x}) \mathrm{d} S(x)-\int_{S} H(\mathbf{s}, \mathbf{x}) p(\mathbf{x}) \mathrm{d} S(\mathbf{x})+q_{\mathrm{inc}}(\mathbf{s}),
$$

where

$$
\begin{gathered}
K(\mathbf{s}, \mathbf{x})=\frac{\partial G(\mathbf{s}, \mathbf{x})}{\partial \mathbf{n}(\mathbf{s})}=-\frac{e^{\mathrm{i} k r}}{4 \pi r^{2}}(1-\mathrm{i} k r) \frac{\partial r}{\partial \mathbf{n}(\mathbf{s})} \\
H(\mathbf{s}, \mathbf{x})=\frac{\partial F(\mathbf{s}, \mathbf{x})}{\partial \mathbf{n}(\mathbf{s})}=\frac{e^{\mathrm{i} k r}}{4 \pi r^{3}}\left[\left(3-3 \mathrm{i} k r-k^{2} r^{2}\right) \frac{\partial r}{\partial \mathbf{n}(\mathbf{s})} \frac{\partial r}{\partial \mathbf{n}(\mathbf{x})}+(1-\mathrm{i} k r) n_{i}(\mathbf{s}) n_{i}(\mathbf{x})\right] .
\end{gathered}
$$

In the above, $q_{\text {inc }}(\mathbf{s})=\frac{\partial p_{\text {inc }}(\mathbf{s})}{\partial \mathbf{n}(\mathbf{s})}$ and $n_{i}$ is the Cartesian component of the normal vector. The Einstein summation convention is used throughout the paper: repeated indices imply a summation over their range.

The use of the CBIE alone for exterior acoustic problems leads to non-uniqueness of the numerical solution at some fictitious eigenfrequencies. The Burton-Miller formulation which is obtained by combining CBIE (Eq. (13)) and HBIE (Eq. (16)) directly can be used to overcome this problem:

$$
\mathrm{CBIE}+\beta \mathrm{HBIE}=0
$$

where $\beta$ is the coupling coefficient, which can be chosen as $\mathrm{i} / k$. In the framework 
of IGABEM, the NURBS basis functions are used to approximate the physics field variables, the sound pressure $p(\xi, \eta)$ and the normal derivative $q(\xi, \eta)$ at a point $(\xi, \eta)$ located on the surface in the parametric space can be expressed as

$$
\begin{aligned}
& p(\xi, \eta)=\sum_{A=1}^{N_{A}} R_{A}(\xi, \eta) \tilde{p}_{A} \\
& q(\xi, \eta)=\sum_{A=1}^{N_{A}} R_{A}(\xi, \eta) \tilde{q}_{A}
\end{aligned}
$$

where $\tilde{p}$ and $\tilde{q}$ are the coefficients associated with the control points to discretize pressure and flux, respectively. Herein, the Greville abscissa are used to obtain the location of the collocation points in the parametric space as

$$
\begin{array}{ll}
\hat{\xi}_{a}=\frac{\xi_{a+1}+\xi_{a+2}+\cdots+\xi_{a+p}}{p}, & a=1, \ldots, n, \\
\hat{\eta}_{b}=\frac{\eta_{b+1}+\eta_{b+2}+\cdots+\eta_{b+l}}{l}, & b=1, \ldots, m .
\end{array}
$$

We use knot vectors to divide the boundary surface in the parametric space. After discretizing the boundary surface, Eq. (13) and Eq. (16) are expressed as

$$
\begin{aligned}
& \frac{1}{2} \sum_{A=1}^{N_{A}} R_{A}\left(\hat{\xi}_{c}, \hat{\eta}_{c}\right) \tilde{p}_{A}=\sum_{e=1}^{N_{e}} \sum_{A=1}^{N_{A}}\left[\int_{\xi_{e}}^{\xi_{e+1}} \int_{\eta_{e}}^{\eta_{e+1}} G\left(\mathbf{s}\left(\hat{\xi}_{c}, \hat{\eta}_{c}\right), \mathbf{x}(\xi, \eta)\right) R_{A}(\xi, \eta) J(\xi, \eta) \mathrm{d} \xi \mathrm{d} \eta\right] \tilde{q}_{A} \\
& -\sum_{e=1}^{N_{e}} \sum_{A=1}^{N_{A}}\left[\int_{\xi_{e}}^{\xi_{e+1}} \int_{\eta_{e}}^{\eta_{e+1}} F\left(\mathbf{s}\left(\hat{\xi}_{c}, \hat{\eta}_{c}\right), \mathbf{x}(\xi, \eta)\right) R_{A}(\xi, \eta) J(\xi, \eta) \mathrm{d} \xi \mathrm{d} \eta\right] \tilde{p}_{A}+p_{\text {inc }}\left(\mathbf{s}_{c}\right),
\end{aligned}
$$

and

$$
\frac{1}{2} \sum_{A=1}^{N_{A}} R_{A}\left(\hat{\xi}_{c}, \hat{\eta}_{c}\right) \tilde{q}_{A}=\sum_{e=1}^{N_{e}} \sum_{A=1}^{N_{A}}\left[\int_{\xi_{e}}^{\xi_{e+1}} \int_{\eta_{e}}^{\eta_{e+1}} K\left(\mathbf{s}\left(\hat{\xi}_{c}, \hat{\eta}_{c}\right), \mathbf{x}(\xi, \eta)\right) R_{A}(\xi, \eta) J(\xi, \eta) \mathrm{d} \xi \mathrm{d} \eta\right] \tilde{q}_{A}
$$


$-\sum_{e=1}^{N_{e}} \sum_{A=1}^{N_{A}}\left[\int_{\xi_{e}}^{\xi_{e+1}} \int_{\eta_{e}}^{\eta_{e+1}} H\left(\mathbf{s}\left(\hat{\xi}_{i}, \hat{\eta}_{j}\right), \mathbf{x}(\xi, \eta)\right) R_{A}(\xi, \eta) J(\xi, \eta) \mathrm{d} \xi \mathrm{d} \eta\right] \tilde{p}_{A}+q_{\mathrm{inc}}\left(\mathbf{s}_{c}\right)$

where $N_{e}$ denotes the number of the discretized NURBS elements, $\left[\xi_{e}, \xi_{e+1}\right] \times\left[\eta_{e}, \eta_{e+1}\right]$ is the $e$-th NURBS element in the parametric space, $J$ is the Jacobian. Then, after collecting the equations for all collocation points and expressing them in matrix form, one can obtain the following system of linear algebraic equations based on the linear combination of Eq. (22) and Eq. (23)

$$
\mathbf{H} \tilde{\mathbf{p}}=\mathbf{G} \tilde{\mathbf{q}}+\mathbf{p}_{\text {inc }}+\beta \mathbf{q}_{\text {inc }} .
$$

In the above equation, $\mathbf{H}$ and $\mathbf{G}$ are matrices constructed from the integrals in Eqs. (22) and (23), $\mathbf{p}$ and $\mathbf{q}$ are vectors containing the acoustic pressure and flux coefficients, and $\mathbf{p}_{\text {inc }}$ and $\mathbf{q}_{\text {inc }}$ are vectors collecting of the pressure and flux of incident wave on collocation points. By solving the above equation, we can obtain the unknown coefficients associated with particular control points. Then using Eq. (13) and setting $c(\mathbf{x})$ as 1, we can obtain the sound pressure in the fluid domain.

\subsection{Evaluation of singular boundary integral}

A main challenge in the implementation of IGABEM is to deal with the integral with various orders of singularity.

\subsubsection{Evaluation of weakly singular boundary integral}

Weakly singular boundary integrals appear in Eq. (22) and Eq. (23) when the source point $\mathbf{x}\left(\hat{\xi}_{c}, \hat{\eta}_{c}\right)$ in parametric space lies within the NURBS element $\left[\xi_{e}, \xi_{e+1}\right] \times$ $\left[\eta_{e}, \eta_{e+1}\right]$, and they need to be treated very carefully. Herein, the singular integrals are evaluated with the method presented by [25] and [58]. First, we define the singular 
boundary integrals in Eq. (22) as follows

$$
\begin{aligned}
& g=\int_{\xi_{e}}^{\xi_{e+1}} \int_{\eta_{e}}^{\eta_{e+1}} G\left(\mathbf{s}\left(\hat{\xi}_{c}, \hat{\eta}_{c}\right), \mathbf{x}(\xi, \eta)\right) R_{A}(\xi, \eta) J(\xi, \eta) \mathrm{d} \xi \mathrm{d} \eta \\
& h=\int_{\xi_{e}}^{\xi_{e+1}} \int_{\eta_{e}}^{\eta_{e+1}} F\left(\mathbf{s}\left(\hat{\xi}_{c}, \hat{\eta}_{c}\right), \mathbf{x}(\xi, \eta)\right) R_{A}(\xi, \eta) J(\xi, \eta) \mathrm{d} \xi \mathrm{d} \eta .
\end{aligned}
$$

The numerical procedure to evaluate the singular integrals in the above two equations is important to the performance and accuracy of the IGABEM. According to the expression of the Green's function, the singularity order of the single layer integral in Eq. (25) is $O(1 / r)$, hence this integral is weakly singular. Although the expression of the derivative of the Green's function contains $1 / r^{2}$, the singularity order of the double layer integral in Eq. (26) is still $O(1 / r)$ because of the existence of $\partial r / \partial \mathbf{n}$. Herein, a polar integration scheme is applied to eliminate the singularity of order $O(1 / r)$, which transfers the integrals in Eqs. (25) and (26) into the format as follows

$$
\begin{aligned}
g^{A} & =\sum_{M_{e}=1}^{4} \int_{\theta_{1}}^{\theta_{2}} \int_{0}^{\rho(\theta)} G\left(\mathbf{s}\left(\hat{\xi}_{c}, \hat{\eta}_{c}\right), \mathbf{x}(\xi, \eta)\right) R_{A}(\xi, \eta) J(\xi, \eta) \rho \mathrm{d} \rho \mathrm{d} \theta \\
h^{A} & =\sum_{M_{e}=1}^{4} \int_{\theta_{1}}^{\theta_{2}} \int_{0}^{\rho(\theta)} F\left(\mathbf{s}\left(\hat{\xi}_{c}, \hat{\eta}_{c}\right), \mathbf{x}(\xi, \eta)\right) R_{A}(\xi, \eta) J(\xi, \eta) \rho \mathrm{d} \rho \mathrm{d} \theta .
\end{aligned}
$$

The quadrilateral element is subdivided into four triangular elements sharing the collocation point as a common vertex, then the Gauss-Legendre integral can be used to solve the integrals in the polar coordinate system.

\subsubsection{Evaluation of hypersingular boundary integral}

However, when the Burton-Miller method is used, the kernel function $H(\mathbf{s}, \mathbf{x})$ in Eq. (16) and Eq. (23) is hypersingular. Herein, the Cauchy principal value and the Hadamard finite part integral method are used to eliminate the singularity. As shown in Fig. 4, for a source point $\mathbf{s}$ located in the boundary $S$, we build a hemispherical region $S_{\varepsilon}^{+}$centered at $\mathbf{s}$ point with a radius $\varepsilon$. $S_{\varepsilon}$ is the subregion of $S$ lying inside the hemispherical region $S_{\varepsilon}^{+}$. The augmented boundary can be approximated as 


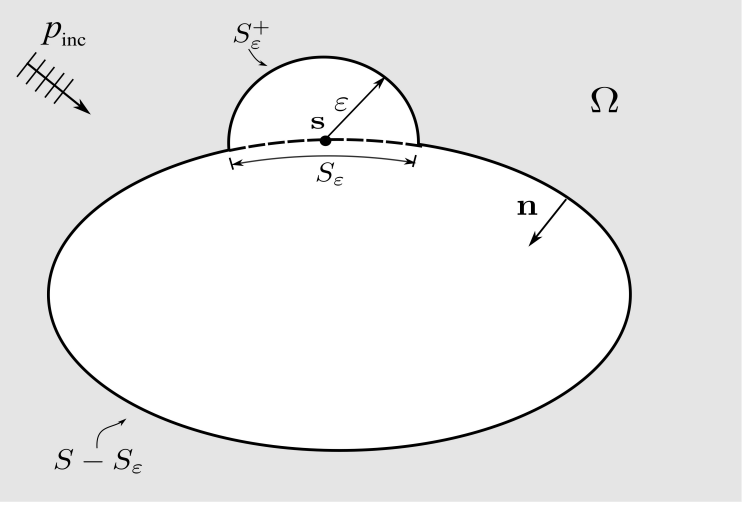

Fig. 4: The source point located on the boundary.

$$
S=\lim _{\varepsilon \rightarrow 0}\left(S-S_{\varepsilon}+S_{\varepsilon}^{+}\right)
$$

We rewrite the hypersingular boundary integral in Eq. (16) according to Eq. (29), as follows

$$
\underbrace{\int_{S} H(\mathbf{s}, \mathbf{x}) p(\mathbf{x}) d S(\mathbf{x})}_{\tilde{g}_{1}}=\underbrace{\lim _{\varepsilon \rightarrow 0} \int_{S-S_{\varepsilon}} H(\mathbf{s}, \mathbf{x}) p(\mathbf{x}) \mathrm{d} S(\mathbf{x})}_{\tilde{g}_{2}}+\underbrace{\lim _{\varepsilon \rightarrow 0} \int_{S_{\varepsilon}^{+}} H(\mathbf{s}, \mathbf{x}) p(\mathbf{x}) \mathrm{d} S(\mathbf{x})}_{\tilde{g}_{3}}
$$

We construct $\tilde{g}^{1}, \tilde{g}^{2}$, and $\tilde{g}^{3}$ to stands for the three integrals in Eq. (30). On $S_{\varepsilon}$, the following formulations hold:

$$
\begin{gathered}
\frac{\partial r}{\partial \mathbf{n}(\mathbf{x})}=1, \\
\frac{\partial r}{\partial \mathbf{n}(\mathbf{s})}=-n_{i}(\mathbf{s}) n_{i}(\mathbf{x}) .
\end{gathered}
$$

Using Eqs. (31) and (32), we can obtain the new expression of $\tilde{g}^{3}$ in the parametric space, as follows

$$
\tilde{g}^{3}=-\frac{1}{2 \pi} \lim _{\varepsilon \rightarrow 0} \int_{0}^{\pi} \frac{n_{i}(\mathbf{s}) n_{i}(\mathbf{x})}{\varepsilon} p(\mathbf{x}) \mathrm{d} \theta=-\frac{1}{2 \pi} \sum_{a=1}^{N_{A}}\left[\lim _{\varepsilon \rightarrow 0} \int_{0}^{\pi} \frac{n_{i}(\mathbf{s}) n_{i}(\mathbf{x})}{\varepsilon} R_{A} \mathrm{~d} \theta\right] \tilde{p}_{A} .
$$


After the use of the NURBS parametric space and the polar coordinate transformation, $\tilde{g}^{2}$ writes

$$
\tilde{g}^{2}=\sum_{a=1}^{N_{A}}\left[\lim _{\varepsilon \rightarrow 0}\left\{\int_{0}^{2 \pi} \int_{\rho_{1}}^{\rho_{2}} H R_{A} J \rho d \rho d \theta\right\}\right] \tilde{p}_{A}=\sum_{A=1}^{N_{A}}\left[\tilde{g}_{f}^{2}\right] \tilde{p}_{A}
$$

We noticed that the hypersingular term in the kernel $H(\mathbf{s}, \mathbf{x})$ is $n_{i}(\mathbf{s}) n_{i}(\mathbf{x}) / 4 \pi r^{3}$. By using the polar coordinate transformation and Taylor's expansion, the hypersingular term is expressed as

$$
f(\rho, \theta)=\frac{n_{i}(\mathbf{s}) n_{i}(\mathbf{x})}{4 \pi r^{3}} R_{A} J \rho=\frac{f_{2}(\theta)}{\rho^{2}}+\frac{f_{1}(\theta)}{\rho}+O\left(\rho^{0}\right) .
$$

Obviously, when $\rho$ is very small, the function $f(\rho, \theta)$ is singular. In order to eliminate the singularity, we remove the singular term as

$$
\begin{aligned}
\tilde{g}_{f}^{2} & =\underbrace{\lim _{\varepsilon \rightarrow 0}\left\{\int_{0}^{2 \pi} \int_{\rho(\varepsilon, \theta)}^{\rho_{2}(\theta)}\left[H R_{A} J \rho-\left(\frac{f_{2}(\theta)}{\rho^{2}}+\frac{f_{1}(\theta)}{\rho}\right)\right] \mathrm{d} \rho \mathrm{d} \theta\right\}}_{I_{0}}+ \\
& \underbrace{\lim _{\varepsilon \rightarrow 0}\left\{\int_{0}^{2 \pi} \int_{\rho(\varepsilon, \theta)}^{\rho_{2}(\theta)} \frac{f_{1}(\theta)}{\rho} \mathrm{d} \rho \mathrm{d} \theta\right\}}_{I_{1}}+\underbrace{\lim _{\varepsilon \rightarrow 0}\left\{\int_{0}^{2 \pi} \int_{\rho(\varepsilon, \theta)}^{\rho_{2}(\theta)} \frac{f_{2}(\theta)}{\rho^{2}} \mathrm{~d} \rho \mathrm{d} \theta\right\}}_{I_{2}} .
\end{aligned}
$$

$I_{0}$ is nonsingular and can be computed using Gauss-Legendre quadrature. However, $I_{1}$ and $I_{2}$ need to be treated specially because of the singularity.

Using a Taylor's expansion, $\rho$ can be expressed as

$$
\rho(\varepsilon)=\varepsilon \beta(\theta)+\varepsilon^{2} \gamma(\theta)+O\left(\varepsilon^{3}\right)
$$

After integration by parts, $I_{1}$ and $I_{2}$ are expressed as

$$
I_{1}=\int_{0}^{2 \pi} f_{1}(\theta) \ln \left|\frac{\rho_{2}(\theta)}{\beta(\theta)}\right| \mathrm{d} \theta
$$




$$
I_{2}=\int_{0}^{2 \pi} f_{2}(\theta)\left[\frac{\gamma(\theta)}{\beta^{2}(\theta)}-\frac{1}{\rho_{2}(\theta)}\right] \mathrm{d} \theta+\lim _{\varepsilon \rightarrow 0}\left[\frac{1}{\varepsilon} \int_{0}^{2 \pi} \frac{f_{2}(\theta)}{\beta(\theta)} \mathrm{d} \theta\right] .
$$

By substituting Eqs. (38), (39), and (36) into Eq. (34), we can obtain the new expression of $\tilde{g}^{2}$ :

$$
\begin{aligned}
\tilde{g}^{2}= & \sum_{A=1}^{N_{a}} \int_{0}^{2 \pi} \int_{0}^{\rho_{2}}\left[H R_{A} J \rho-\left(\frac{f_{2}(\theta)}{\rho^{2}}+\frac{f_{1}(\theta)}{\rho}\right)\right] \mathrm{d} \rho \mathrm{d} \theta \tilde{p}_{A}+ \\
& \sum_{a=1}^{N_{A}} \int_{0}^{2 \pi} f_{1}(\theta) \ln \left|\frac{\rho_{2}(\theta)}{\beta(\theta)}\right| \mathrm{d} \theta \tilde{p}_{A}+\sum_{a=1}^{N_{A}} \int_{0}^{2 \pi} f_{2}(\theta)\left[\frac{\gamma(\theta)}{\beta^{2}(\theta)}-\frac{1}{\rho_{2}(\theta)}\right] \mathrm{d} \theta \tilde{p}_{A}+ \\
& \sum_{a=1}^{N_{A}} \lim _{\varepsilon \rightarrow 0}\left[\frac{\tilde{p}_{A}}{\varepsilon} \int_{0}^{2 \pi} \frac{f_{2}(\theta)}{\beta(\theta)} \mathrm{d} \theta\right] .
\end{aligned}
$$

We notice that the last term in Eq. (40) and the term in the right hand side of Eq. (33) are both singular. According to Lyapunov-Tauber theory, the two singular terms must cancel each other. So, the non-singular formulation of $\tilde{g}^{1}$ is expressed as

$$
\begin{aligned}
\tilde{g}^{1}= & \sum_{a=1}^{N_{A}} \int_{0}^{2 \pi} \int_{0}^{\rho_{2}}\left[H R_{A} J \rho-\left(\frac{f_{2}(\theta)}{\rho^{2}}+\frac{f_{1}(\theta)}{\rho}\right)\right] \mathrm{d} \rho \mathrm{d} \theta \tilde{p}_{A}+ \\
& \sum_{a=1}^{N_{A}} \int_{0}^{2 \pi} f_{1}(\theta) \ln \left|\frac{\rho_{2}(\theta)}{\beta(\theta)}\right| \mathrm{d} \theta \tilde{p}_{A}+\sum_{A=1}^{N_{A}} \int_{0}^{2 \pi} f_{2}(\theta)\left[\frac{\gamma(\theta)}{\beta^{2}(\theta)}-\frac{1}{\rho_{2}(\theta)}\right] \mathrm{d} \theta \tilde{p}_{A}
\end{aligned}
$$

We now need to compute the coefficient $f_{1}(\theta), f_{2}(\theta), \beta(\theta)$, and $\gamma(\theta)$. Please refer to Appendix A for details.

\section{IGABEM for shape sensitivity and optimization analysis}

\subsection{IGABEM sensitivity formulation with Burton-Miller method}

Sensitivity analysis refers to the evaluation of derivatives of the objective functions with respect to design variables. This is a critical step for gradient-based optimization analysis. Direct differentiation methods (DDM), which rely on a direct differentiation of the boundary integral equations with respect to design variables, generate analytical formulations of sensitivity of objective function. Due to its accuracy and convenience 
for BEM, the present work will apply this direct differentiation method to calculate the sensitivity of the objective function.

By differentiating Eqs. (13) and (16) with respect to design variables, we can obtain the following sensitivity formulas

$$
\begin{aligned}
c(\mathbf{s}) \dot{p}(\mathbf{s})= & \int_{S}[\dot{G}(\mathbf{s}, \mathbf{x}) q(\mathbf{x})-\dot{F}(\mathbf{s}, \mathbf{x}) p(\mathbf{x})] \mathrm{d} S(\mathbf{x}) \\
& +\int_{S}[G(\mathbf{s}, \mathbf{x}) \dot{q}(\mathbf{x})-F(\mathbf{s}, \mathbf{x}) \dot{p}(\mathbf{x})] \mathrm{d} S(\mathbf{x}) \\
& +\int_{S}[G(\mathbf{s}, \mathbf{x}) q(\mathbf{x})-F(\mathbf{s}, \mathbf{x}) p(\mathbf{x})] \mathrm{d} \dot{S}(\mathbf{x})+\dot{p}_{\mathrm{inc}}(\mathbf{s}),
\end{aligned}
$$

and

$$
\begin{aligned}
c(\mathbf{s}) \dot{q}(\mathbf{s})= & \int_{S}[\dot{K}(\mathbf{s}, \mathbf{x}) q(\mathbf{x})-\dot{H}(\mathbf{s}, \mathbf{x}) p(\mathbf{x})] \mathrm{d} S(\mathbf{x}) \\
& +\int_{S}[K(\mathbf{s}, \mathbf{x}) \dot{q}(\mathbf{x})-H(\mathbf{s}, \mathbf{x}) \dot{p}(\mathbf{x})] \mathrm{d} S(\mathbf{x}) \\
& +\int_{S}[K(\mathbf{s}, \mathbf{x}) q(\mathbf{x})-H(\mathbf{s}, \mathbf{x}) p(\mathbf{x})] \mathrm{d} \dot{S}(\mathbf{x})+\dot{q}_{\mathrm{inc}}(\mathbf{s}),
\end{aligned}
$$

where $(\dot{)}$ denotes the derivative of functions with respect to design variables. The sensitivity formulas of the kernel functions with respect to design variables are derived by

$$
\begin{aligned}
\dot{G}(\mathbf{s}, \mathbf{x}) & =-\frac{\mathrm{e}^{\mathrm{i} k r}}{4 \pi r^{2}}(1-\mathrm{i} k r) \dot{r}, \\
\dot{F}(\mathbf{s}, \mathbf{x}) & =\frac{\mathrm{e}^{\mathrm{i} k r}}{4 \pi r^{3}}\left[\left(2-2 \mathrm{i} k r-k^{2} r^{2}\right) \frac{\partial r}{\partial \mathbf{n}(\mathbf{x})} \dot{r}-(1-\mathrm{i} k r) r\left(\frac{\dot{\partial} r}{\partial \mathbf{n}(\mathbf{x})}\right)\right], \\
\dot{K}(\mathbf{s}, \mathbf{x}) & =\frac{\mathrm{e}^{\mathrm{i} k r}}{4 \pi r^{3}}\left[\left(2-2 \mathrm{i} k r-k^{2} r^{2}\right) \frac{\partial r}{\partial \mathbf{n}(\mathbf{s})} \dot{r}-(1-\mathrm{i} k r) r\left(\frac{\dot{\partial} r}{\partial \mathbf{n}(\mathbf{s})}\right)\right], \\
\dot{H}(\mathbf{s}, \mathbf{x}) & =\frac{\mathrm{e}^{\mathrm{i} k r}}{4 \pi r^{4}}\left(-9+9 \mathrm{i} k r+4 k^{2} r^{2}-\mathrm{i} k^{3} r^{3}\right) \frac{\partial r}{\partial \mathbf{n}(\mathbf{s})} \frac{\partial r}{\partial \mathbf{n}(\mathbf{x})} \dot{r} \\
& -\frac{\mathrm{e}^{\mathrm{i} k r}}{4 \pi r^{4}}\left(3-3 \mathrm{i} k r-k^{2} r^{2}\right) n_{l}(\mathbf{s}) n_{l}(\mathbf{x}) \dot{r}
\end{aligned}
$$




$$
\begin{aligned}
& +\frac{\mathrm{e}^{\mathrm{i} k r}}{4 \pi r^{3}}\left(3-3 \mathrm{i} k r-k^{2} r^{2}\right)\left[\left(\frac{\dot{\partial} r}{\partial \mathbf{n}(\mathbf{s})}\right) \frac{\partial r}{\partial \mathbf{n}(\mathbf{x})}+\frac{\partial r}{\partial \mathbf{n}(\mathbf{s})}\left(\frac{\dot{\partial} r}{\partial \mathbf{n}(\mathbf{x})}\right)\right] \\
& +\frac{\mathrm{e}^{\mathrm{i} k r}}{4 \pi r^{3}}(1-\mathrm{i} k r)\left(\dot{n}_{l}(\mathbf{x}) n_{l}(\mathbf{x})+n_{l}(\mathbf{x}) \dot{n}_{l}(\mathbf{x})\right),
\end{aligned}
$$

where the sensitivity formulas of some functions with respect to design variables in the above four equations are derived by

$$
\begin{gathered}
\dot{r}=r_{, i}\left(\dot{x}_{i}-\dot{s}_{i}\right)=\frac{x_{i}-s_{i}}{r}\left(\dot{x}_{i}-\dot{s}_{i}\right), \\
\left(\frac{\dot{\partial} r}{\partial \mathbf{n}}\right)=\left(\dot{r_{, i} n_{i}}\right)=\left(\dot{r_{, i}}\right) n_{i}+r_{, i} \dot{n}_{i}, \\
\left(\dot{r_{, i}}\right)=\left(\frac{x_{i}-s_{i}}{r}\right)=\frac{\left(\dot{x}_{i}-\dot{s}_{i}\right) r-\left(x_{i}-s_{i}\right) \dot{r}}{r^{2}} .
\end{gathered}
$$

The sensitivity of the unit outward normal $n_{i}$ on the boundary surface is

$$
\dot{n}_{i}=\left[\frac{J_{i}(\dot{\xi}, \eta)}{J(\xi, \eta)}\right]=\frac{\dot{J}_{i}(\xi, \eta) J(\xi, \eta)-J_{i}(\xi, \eta) \dot{J}(\xi, \eta)}{J^{2}(\xi, \eta)}
$$

where

$$
\dot{J}(\xi, \eta)=\frac{\dot{J}_{i}(\xi, \eta) J_{i}(\xi, \eta)}{J(\xi, \eta)}
$$

and

$$
\begin{aligned}
& J_{1}(\xi, \eta)=-\frac{\partial x_{2}}{\partial \xi} \frac{\partial x_{3}}{\partial \eta}+\frac{\partial x_{2}}{\partial \eta} \frac{\partial x_{3}}{\partial \xi}, \\
& J_{2}(\xi, \eta)=-\frac{\partial x_{3}}{\partial \xi} \frac{\partial x_{1}}{\partial \eta}+\frac{\partial x_{3}}{\partial \eta} \frac{\partial x_{1}}{\partial \xi}, \\
& J_{3}(\xi, \eta)=-\frac{\partial x_{1}}{\partial \xi} \frac{\partial x_{2}}{\partial \eta}+\frac{\partial x_{1}}{\partial \eta} \frac{\partial x_{2}}{\partial \xi} .
\end{aligned}
$$


By differentiating Eq. (11) with respect to design variables, we obtain the sensitivity formulas of the coordinates at any point located on the boundary surface, as follows

$$
\dot{\mathbf{x}}(\xi, \eta)=\sum_{A=1}^{N_{A}} R_{A}(\xi, \eta) \dot{\mathbf{P}}_{A}
$$

Similarly, according to the above formulas, the following expressions can also be derived:

$$
\begin{aligned}
& {\left[\frac{\partial}{\partial \xi} \mathbf{x}(\xi, \eta)\right]=\sum_{A=1}^{N_{A}} \frac{\partial R_{A}(\xi, \eta)}{\partial \xi} \dot{\mathbf{P}}_{A},} \\
& {\left[\frac{\partial}{\partial \eta} \mathbf{x}(\xi, \eta)\right]=\sum_{A=1}^{N_{A}} \frac{\partial R_{A}(\xi, \eta)}{\partial \eta} \dot{\mathbf{P}}_{A} .}
\end{aligned}
$$

When NURBS basis functions are used to approximate the physics, the sensitivity of the pressure and its normal flux at any points on the boundary surface is obtained by differentiating Eq. (20) with respect to design variables:

$$
\begin{gathered}
\dot{p}(\xi, \eta)=\sum_{A=1}^{N_{A}} R_{A}(\xi, \eta) \dot{\tilde{p}}_{A} \\
\dot{q}(\xi, \eta)=\sum_{A=1}^{N_{A}} R_{A}(\xi, \eta) \dot{\tilde{q}}_{A}
\end{gathered}
$$

Again, when the boundary at point $x$ is smooth, Eqs. (42) and (43) are rewritten as

$$
\begin{aligned}
\frac{1}{2} \sum_{A=1}^{N_{A}} R_{A}\left(\hat{\xi}_{c}, \hat{\eta}_{c}\right) \dot{\tilde{p}}_{A}= & \sum_{e=1}^{N_{e}} \sum_{A=1}^{N_{A}} \int_{\xi_{e}}^{\xi_{e+1}} \int_{\eta_{e}}^{\eta_{e+1}}\left[\dot{G}\left(\mathbf{s}\left(\hat{\xi}_{c}, \hat{\eta}_{c}\right), \mathbf{x}(\xi, \eta)\right) \tilde{q}_{A}-\right. \\
& \left.\dot{F}\left(\mathbf{s}\left(\hat{\xi}_{c}, \hat{\eta}_{c}\right), \mathbf{x}(\xi, \eta)\right) \tilde{p}_{A}\right] R_{A}(\xi, \eta) J(\xi, \eta) \mathrm{d} \xi \mathrm{d} \eta+ \\
& \sum_{e=1}^{N_{e}} \sum_{A=1}^{N_{A}} \int_{\xi_{e}}^{\xi_{e+1}} \int_{\eta_{e}}^{\eta_{e+1}}\left[G\left(\mathbf{s}\left(\hat{\xi}_{i}, \hat{\eta}_{j}\right), \mathbf{x}(\xi, \eta)\right) \dot{\tilde{q}}_{A}-\right. \\
& \left.F\left(\mathbf{s}\left(\hat{\xi}_{c}, \hat{\eta}_{c}\right), \mathbf{x}(\xi, \eta)\right) \dot{\tilde{p}}_{A}\right] R_{A}(\xi, \eta) J(\xi, \eta) \mathrm{d} \xi \mathrm{d} \eta+ \\
& \sum_{e=1}^{N_{e}} \sum_{A=1}^{N_{A}} \int_{\xi_{e}}^{\xi_{e+1}} \int_{\eta_{e}}^{\eta_{e+1}}\left[G\left(\mathbf{s}\left(\hat{\xi}_{i}, \hat{\eta}_{j}\right), \mathbf{x}(\xi, \eta)\right) \tilde{q}_{A}-\right.
\end{aligned}
$$




$$
\left.F\left(\mathbf{s}\left(\hat{\xi}_{c}, \hat{\eta}_{c}\right), \mathbf{x}(\xi, \eta)\right) \tilde{p}_{A}\right] R_{A}(\xi, \eta) \dot{J}(\xi, \eta) \mathrm{d} \xi \mathrm{d} \eta+\dot{p}_{\mathrm{inc}}\left(\mathbf{s}_{c}\right)
$$

and

$$
\begin{aligned}
\frac{1}{2} \sum_{A=1}^{N_{A}} R_{A}\left(\hat{\xi}_{c}, \hat{\eta}_{c}\right) \dot{\tilde{q}}_{A}= & \sum_{e=1}^{N_{e}} \sum_{A=1}^{N_{A}} \int_{\xi_{e}}^{\xi_{e+1}} \int_{\eta_{e}}^{\eta_{e+1}}\left[\dot{K}\left(\mathbf{s}\left(\hat{\xi}_{i}, \hat{\eta}_{j}\right), \mathbf{x}(\xi, \eta)\right) \tilde{q}_{A}-\right. \\
& \left.\dot{H}\left(\mathbf{s}\left(\hat{\xi}_{c}, \hat{\eta}_{c}\right), \mathbf{x}(\xi, \eta)\right) \tilde{p}_{A}\right] R_{a}(\xi, \eta) J(\xi, \eta) \mathrm{d} \xi \mathrm{d} \eta+ \\
& \sum_{e=1}^{N_{e}} \sum_{A=1}^{N_{A}} \int_{\xi_{e}}^{\xi_{e+1}} \int_{\eta_{e}}^{\eta_{e+1}}\left[K\left(\mathbf{s}\left(\hat{\xi}_{c}, \hat{\eta}_{c}\right), \mathbf{x}(\xi, \eta)\right) \dot{\tilde{q}}_{A}-\right. \\
& \left.H\left(\mathbf{s}\left(\hat{\xi}_{c}, \hat{\eta}_{c}\right), \mathbf{x}(\xi, \eta)\right) \dot{\tilde{p}}_{A}\right] R_{A}(\xi, \eta) J(\xi, \eta) \mathrm{d} \xi \mathrm{d} \eta+ \\
& \sum_{e=1}^{N_{e}} \sum_{A=1}^{N_{A}} \int_{\xi_{e}}^{\xi_{e+1}} \int_{\eta_{e}}^{\eta_{e+1}}\left[K\left(\mathbf{s}\left(\hat{\xi}_{c}, \hat{\eta}_{c}\right), \mathbf{x}(\xi, \eta)\right) \tilde{q}_{A}-\right. \\
& \left.H\left(\mathbf{s}\left(\hat{\xi}_{c}, \hat{\eta}_{c}\right), \mathbf{x}(\xi, \eta)\right) \tilde{p}_{A}\right] R_{A}(\xi, \eta) \dot{J}(\xi, \eta) \mathrm{d} \xi \mathrm{d} \eta++\dot{q}_{\mathrm{inc}}\left(\mathbf{s}_{c}\right) .
\end{aligned}
$$

After collecting the equations for all collocation points and expressing them in matrix form, we obtain a system of linear algebraic equations based on the linear combination of Eq. (58) and Eq. (59):

$$
\dot{\mathbf{H}} \tilde{\mathbf{p}}+\mathbf{H} \dot{\tilde{\mathbf{p}}}=\dot{\mathbf{G}} \tilde{\mathbf{q}}+\mathbf{G} \dot{\tilde{\mathbf{q}}}+\dot{\mathbf{p}}_{\text {inc }}+\beta \dot{\mathbf{q}}_{\text {inc }}
$$

By solving the above equations, we can obtain the unknown sensitivity values at particular control points. Using Eq. (42) and setting $c(\mathbf{x})$ to be 1 , we obtain the sound pressure sensitivity in the fluid domain. Similarly to section 3.2, singular boundary integrals appear in Eq. (42) and Eq. (43) when the source point $\mathbf{x}\left(\hat{\xi}_{i}, \hat{\eta}_{j}\right)$ in the parametric space lies on the NURBS element $\left[\xi_{e}, \xi_{e+1}\right] \times\left[\eta_{e}, \eta_{e+1}\right]$. Those need to be treated very carefully (see Appendix B). 


\subsection{Shape optimization analysis with IGABEM}

In this section, we apply the IGABEM to gradient-based shape optimization in structural-acoustic problems. The associated mathematical model can be expressed as

$$
\begin{cases}\min & \Pi\left(\tau_{I}, \mathbf{p}\left(\tau_{I}\right)\right), \\ \text { s.t. } & V\left(\tau_{I}\right)-V_{0} \leqslant 0, \\ & \tau_{I}^{\min } \leqslant \tau_{I} \leqslant \tau_{I}^{\max }\end{cases}
$$

The symbol $\Pi$ stands for the objective function and $\mathbf{p}$ the vector collecting the sound pressure at a series of points in the reference zone. $\tau_{I}$ denotes the shape design variable with index $I$, which varies between the lower bound $\tau_{I}^{\min }$ and the upper bound $\tau_{I}^{\max }$. In IGABEM, a natural choice of the design variables is the Cartesian coordinates of the control points. $V_{0}$ is a specified value for setting the maximum volume constraint, and $V\left(\tau_{I}\right)$ is the volume of the structure which can be calculated as follows

$$
V=\int_{\Omega} \mathrm{d} \Omega=\frac{1}{3} \sum_{e=1}^{n_{e}} \int_{0}^{1} \int_{0}^{1} \mathbf{x} \cdot \mathbf{n} J_{e}(\xi, \eta) \mathrm{d} \xi \mathrm{d} \eta
$$

For gradient-based shape optimization, it is essential to evaluate the shape derivatives of the objective function

$$
\frac{\mathrm{d} \Pi}{\mathrm{d} \tau_{I}}=\frac{\partial \Pi}{\partial \tau_{I}}+\frac{\partial \Pi}{\partial \mathbf{p}} \frac{\partial \mathbf{p}}{\partial \tau_{I}}
$$

where $\frac{\partial \mathbf{p}}{\partial \tau_{I}}$ can be evaluated using the technique detailed in Section 4.1. Additionally, the volume constraint function should be calculated as:

$$
\frac{\mathrm{d} V}{\mathrm{~d} \tau_{I}}=\frac{1}{3} \sum_{e=1}^{n_{e}} \int_{0}^{1} \int_{0}^{1}\left[\frac{\partial \mathbf{x}}{\partial \tau_{I}} \cdot \mathbf{n} J_{e}(\xi, \eta)+\mathbf{x} \cdot \frac{\partial \mathbf{n}}{\partial \tau_{I}} J_{e}(\xi, \eta)+\mathbf{x} \cdot \mathbf{n} \frac{\partial \mathbf{J}_{\mathbf{e}}(\xi, \eta)}{\partial \tau_{I}}\right] \mathrm{d} \xi \mathrm{d} \eta
$$

We use the method of moving asymptotes (MMA) [60] as the optimizer to update the design variables in each iteration step until the objective function is converged. 


\section{Numerical examples}

Numerical examples are presented in this section to verify the proposed algorithm and show its capability for practical engineering problems. The solution procedure for the numerical analysis is implemented using Fortran 95 with OpenMP parallelization. The shape optimization examples are exterior acoustic scattering problems, so the term related to incidence wave is kept in the boundary integral equations. The objective function is chosen as $\Pi=\mathbf{p}^{\mathrm{H}} \mathbf{p}$, where $\mathbf{p}$ denotes the sound pressure at the points within

a reference zone and ()$^{\mathrm{H}}$ the conjugate transpose of a vector. Correspondingly, the shape derivative of the objective function is

$$
\frac{\partial \Pi}{\partial \tau_{I}}=\frac{\partial\left(\mathbf{p}^{\mathrm{H}} \mathbf{p}\right)}{\partial \tau_{I}}=2 \Re\left(\mathbf{p}^{\mathrm{H}} \frac{\partial \mathbf{p}}{\partial \tau_{I}}\right)
$$

\subsection{Sphere model}

In this subsection, the model of the spherical shell with a radius $r=1.0 \mathrm{~m}$ is considered. Fig. 5 shows its NURBS surface description and the corresponding control grid. The fluid is the air with density $\rho_{f}=1.2 \mathrm{~kg} / \mathrm{m}^{3}$, and the speed of the acoustic wave in the air is $c=343 \mathrm{~m} / \mathrm{s}$.

\subsubsection{Pulsating sphere}

Before we proceed to conduct shape optimization, a pulsating spherical surface example is adopted to verify the acoustic analysis and shape sensitivity analysis of the present algorithm. In this example, the Neumann boundary condition is used. The relation between the vibration velocity of the spherical sphere $v_{n}$ and the pressure flux $q_{0}$ is $q_{0}=\mathrm{i} \omega \rho_{f} v_{n}$. The pressure flux $q_{0}$ on the spherical shell is set as 1509.6i. Since numerical errors mainly come from physical field discretization and numerical integration, the corresponding convergence studies are performed to better understand the performance of the proposed algorithm. Fig. 6a shows that the relative error between the numerical results and the analytical solutions decreases with the increase of degrees of freedom 


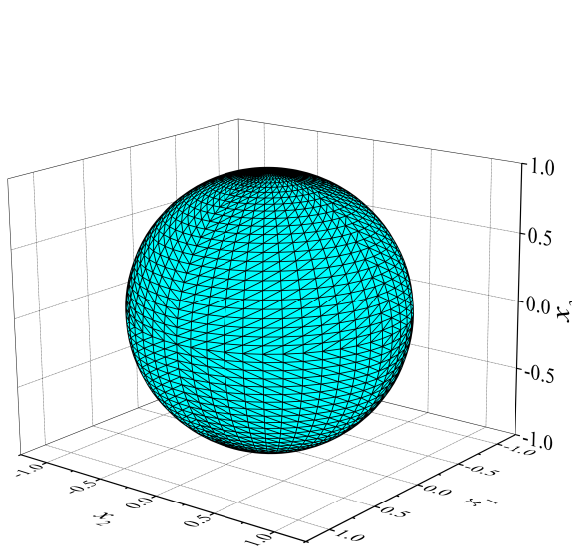

(a) NURBS model of pulsating sphere

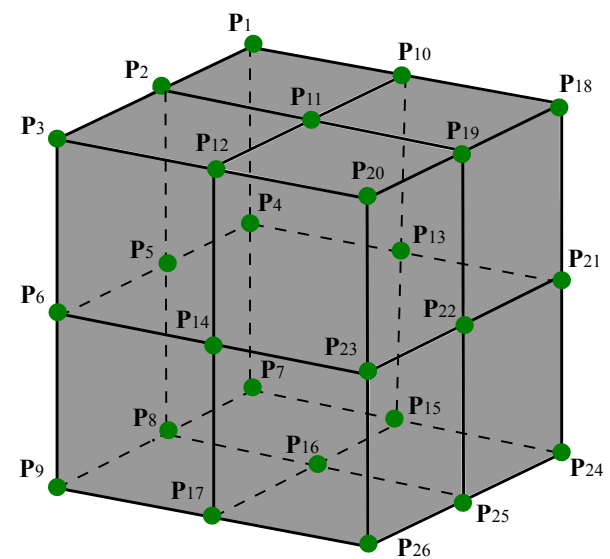

(b) Control points and design variables distribution

Fig. 5: Two knot vectors in two dimension are $\Xi=[0,0,0,0.5,0.5,1,1,1]$ and $\Upsilon=$ $[0,0,0,0.25,0.25,0.5,0.5,0.75,0.75,1,1,1]$, respectively; The order is $p=2$ and $l=2$; The number of the control points is 27; The coordinates of the control point are $(0,0,1)$ for $\mathbf{P}_{11},(0,1,0)$ for $\mathbf{P}_{22},(0,-1,0)$ for $\mathbf{P}_{5}$, and $(0,0,-1)$ for $\mathbf{P}_{16}$.

(DOFs). Fig. $6 \mathrm{~b}$ plots the relative error in terms of the number of Gauss quadrature points. The error first decreases rapidly and then converges as Gauss quadrature points increase. Compared to conventional BEM based on Lagrange polynomials, the high order NURBS basis functions in IGABEM improve the approximation accuracy of physical fields, but require more quadrature points.

Now we turn to the verification of shape sensitivity analysis, which plays a key role in shape optimization. Herein, we choose the sound pressure at a point $(2,0,0)$ as the objective function and set the design variable as the $x$-coordinate of the control point $\mathbf{P}_{21}$, which varies from 0.5 to 1.5 . With the change of the design variable, the structural model evolves to irregular shape from a sphere. We calculate the acoustic pressure shape sensitivity for a series of geometries formed in this shape morphing procedure. We make a comparison between the sensitivity analysis solutions evaluated by direct differentiation methods and finite difference methods. As shown in Fig. 7, the results of direct differentiation methods and finite difference methods are in good agreement, which verifies the algorithm proposed in this paper. 


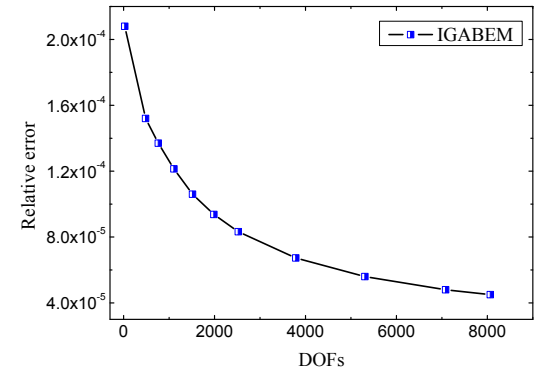

(a) Relative error in terms of DOFs

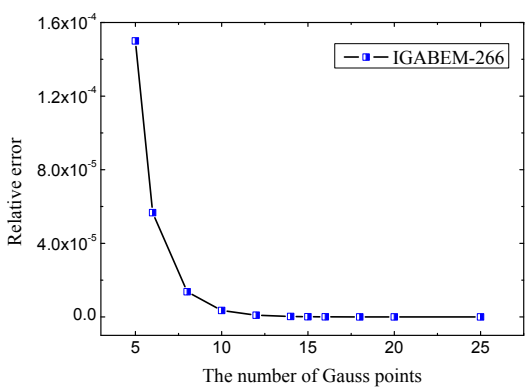

(b) Relative error in terms of Gauss points

Fig. 6: The relative error between numerical results of sound pressure at a point $(2,0,0)$ and analytical results is computed at the frequency $100 \mathrm{~Hz}$ : (a) $5 \times 5$ Gauss points are used; (b) The number of elements and collocation points are 72 and 266, respectively. The $x$-coordinate stands for the number of Gauss points. For example 10 in $x$ axis means $10 \times 10$ Gauss points.

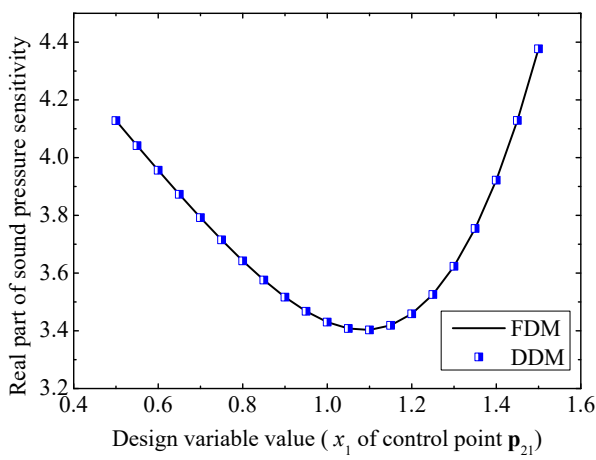

(a) Real part

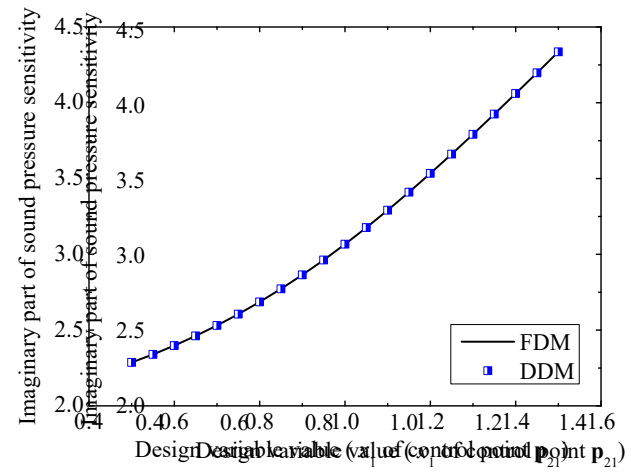

(b) Imaginary part

Fig. 7: Sound pressure sensitivity at a point $(2,0,0)$ : The design variable is set to be the $x$-coordinate of control point $\mathbf{P}_{21}$ at the frequency $100 \mathrm{~Hz}$; The lower bound and the upper bound of the design variable are set to be 0.5 and 1.5, respectively; FDM means the results from the finite difference method, and DDM the direct differentiation method.

To gain insights into the effect of geometries on physical fields, we create three different geometries by modifying the position of control point $\mathbf{P}_{22}$ and/or $\mathbf{P}_{5}$ of the sphere model. They are named as "Shape 1","Shape 2", and "Shape 3", respectively, as depicted in Fig. 8(a,d,g). The element number is 128 and the number of the collocation points is 482 . The average number of elements per wave length at $100 \mathrm{~Hz}$ is more than $30.8 \times 8$ Gauss points are used for regular boundary integration, and 
$15 \times 15$ for irregular boundary integration. The sound pressure contour on the spherical surface with a radius $2 \mathrm{~m}$ around the three geometries is plotted in Fig. 8, where the Neumann boundary condition with the same pressure flux is used and the computing frequency is $100 \mathrm{~Hz}$. It is evident that the three different shapes exhibit completely different distribution of the real part and imaginary part of the sound pressure. Furthermore, a sensitivity analysis with respect to the coordinates of the control point $\mathbf{P}_{22}$ in $y$-axis is conducted for the three geometries, respectively. As illustrated in Fig. 9, the sensitivity of the sound pressure in the region approaching control point $\mathbf{P}_{16}$ is larger than the sensitivity further away from the control point. This example demonstrates the advantage of the IGA in structural modeling and numerical analysis, in particular for shape optimization analysis, because altering the control points is flexible to generate various shapes during optimization without requiring any meshing or remeshing at any time.

\subsubsection{Plane wave scattering by a sphere}

Now we perform shape optimization analysis for the spherical geometry in Fig. 5 as an initial shape. An incident plane wave with a unit amplitude is traveling along the positive $y$ axis and several different frequencies are used in the test. The nine control points $\mathbf{P}_{1}-\mathbf{P}_{9}$ with minimum coordinate value -1 in $y$ axis are set as design control points (Fig. 5b). To reduce the complexity, only the coordinates on the $y$ axis of the nine control points are chosen as design variables, and they are subject to the same side constraints when they are moving. The lower bound and the upper bound of the design variables are -1.5 and -0.5 , respectively. For ease of visualizing the solution of the objective function, a series of points are selected lying on a line perpendicular to the $x-y$ plane. The projection of this line on the $x y$ plane is a point with coordinates $(0,2)$ in xoy coordinate system. The length of the line is $2 \mathrm{~m}$ and the $z$-coordinate of the middle point of this line is 0 . In total 21 points are considered in the line perpendicular to the $x-y$ plane to calculate the objective function. 

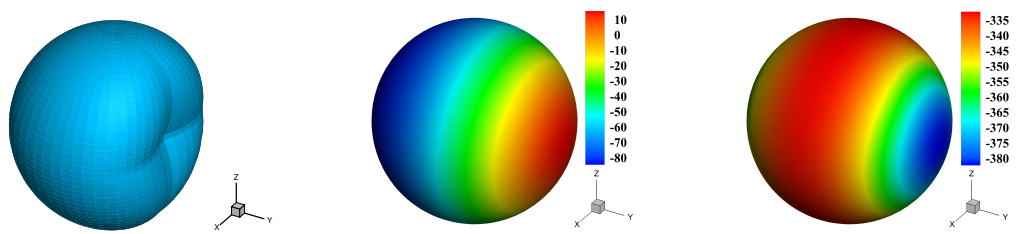

(a) Shape 1

(b) Real part of pressure for shape(c) Imaginary part of pressure for shape 1
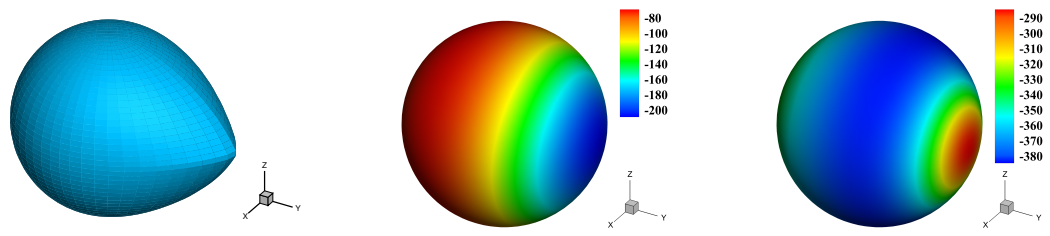

(d) Shape 2

(e) Real part of pressure for shape(f) Imaginary part of pressure for 2 shape 2
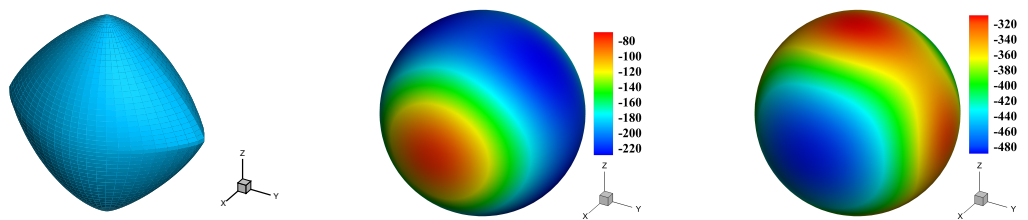

(g) Shape 3

(h) Real part of pressure for shape(i) Imaginary part of pressure for 3 shape 3

Fig. 8: The structure models evolved from a sphere and the sound pressure contour on a spherical surface with a radius $2 \mathrm{~m}$ : (a) "Shape 1" is constructed by changing the coordinate of the control point $\mathbf{P}_{22}$ from $(0,1,0)$ to $(0,0.5,0)$ and keeping the other parameters unchanged; (b) and (c) show the distribution of the real part and imaginary part of the sound pressure for shape 1, respectively; (d) "Shape 2" is constructed by changing the coordinate of the control point $\mathbf{P}_{22}$ to $(0,1.5,0)$; (e) and (f) show the distribution of the real part and imaginary part of the sound pressure for shape 2, respectively; (g) "Shape 3" is constructed by changing the coordinate of three control points, such as $(0,1.5,0)$ for $\mathbf{P}_{22},(0,-1.5,0)$ for $\mathbf{P}_{5}$, and $(0,0,1.5)$ for $\mathbf{P}_{11}$; (h) and (i) show the distribution of the real part and imaginary part of the sound pressure for shape 3 , respectively.

The start values of the objective function before optimization changes with frequencies. Please see Fig. 10. We can find that the solution with 114 DOFs deviates greatly from the analytical solution when the frequency is more than $160 \mathrm{~Hz}$, and the 

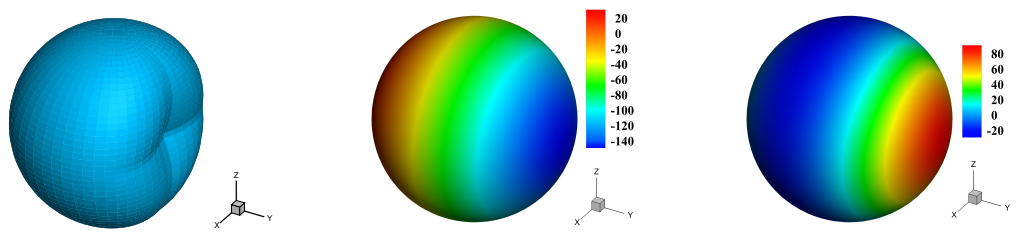

(a) Shape 1

(b) Real part of pressure sensitiv-(c) Imaginary part of pressure sen-

ity sitivity
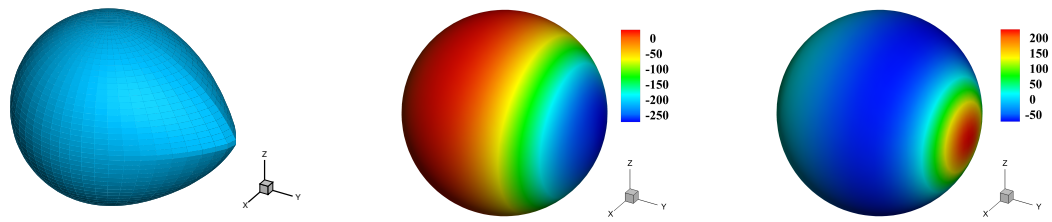

(d) Shape 2

(e) Real part of pressure sensitiv-(f) Imaginary part of pressure senity sitivity
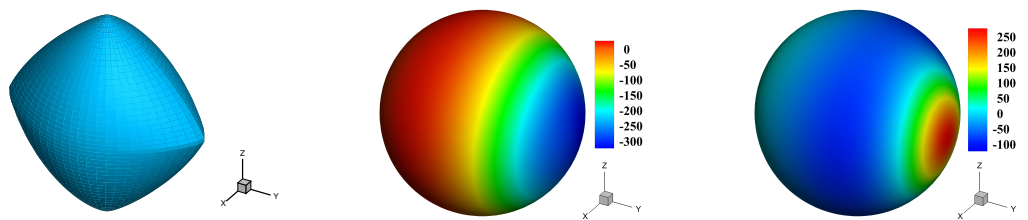

(g) Shape 3

(h) Real part of pressure sensitiv-(i) Imaginary part of pressure senity sitivity

Fig. 9: The structure models evolved from a sphere and the sound pressure sensitivity contour on a spherical surface with a radius $2 \mathrm{~m}$ : (b) and (c) show the distribution of the real part and imaginary part of the sound pressure sensitivity for shape 1; (e) and (f) for shape 2; respectively; (h) and (i) for shape 3.

solution with 266 DOFs deviates greatly from the analytical solution when the frequency is more than $300 \mathrm{~Hz}$. In comparison, the results of IGABEM with $482 \mathrm{DOFs}$ are in good agreement with the analytical solutions, although there is a slight deviation between them after $300 \mathrm{~Hz}$. Thus, the higher the computational frequency is, the more refined meshes and degrees of freedom are needed. However, computational efficiency of the IGABEM based on NURBS decreases rapidly with the increase of DOF. It is 


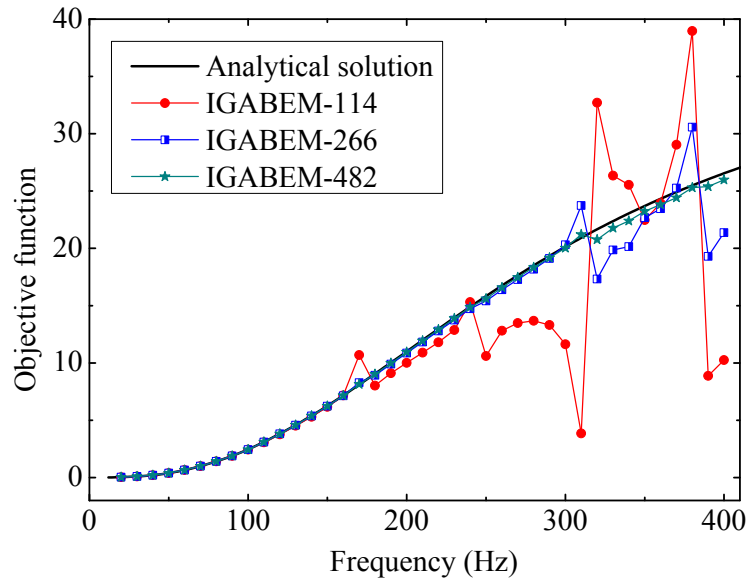

Fig. 10: The objective function in terms of frequencies with different DOFs.

worth noting that NURBS basis functions are evaluated using recursion formula, so more computation time for IGABEM is required than conventional BEM. When the number of DOFs is set to 482, the computation time for IGABEM is 110 seconds, but 20 seconds for conventional BEM. When the DOFs is set to 1106 , the computation time for IGABEM is 959 seconds, but 50 seconds for conventional BEM. Therefore, IGABEM consumes more computation time than conventional BEM, although such problem can be remedied by Bézier extraction. On the other hand, IGABEM achieves higher accuracy than conventional BEM with the same DOFs.

In this subsection, a NURBS sphere model with 266 DOFs is used in shape optimization. After the iterative procedure (Fig. 11), the optimized design is reached and the final geometry shown in Fig. 12. The optimization objective in Fig. 11(a) reduces to around $90 \%$ at $100 \mathrm{~Hz}$. The constraint function in Fig. 11(b) first decreases, then increases, and finally approaches the maximum constraint value. The final optimized design variables can be seen in Table 1. It demonstrates the potential of this proposed algorithm in shape optimization. 


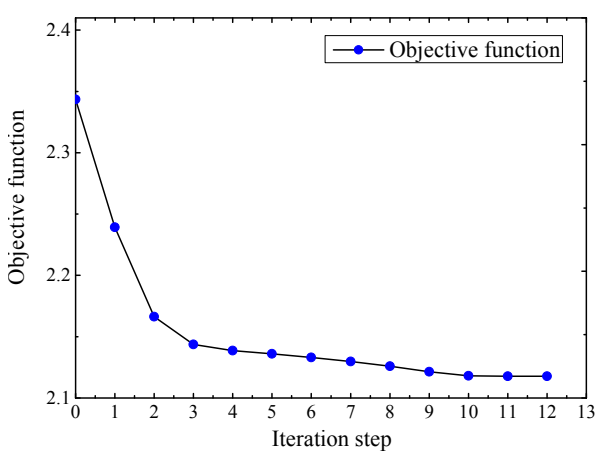

(a) Objective function in terms of iteration step

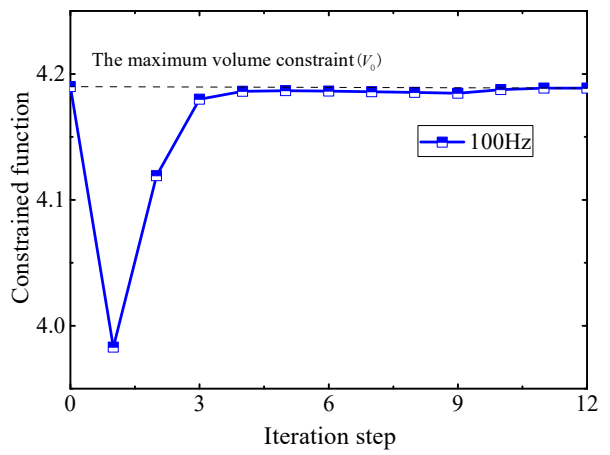

(b) Constraint function in terms of iteration step

Fig. 11: The convergence of the iterative process for the scattering sphere optimization problem at $100 \mathrm{~Hz}$.

Table 1: Design variables in the sphere optimization procedure at $100 \mathrm{~Hz}$

\begin{tabular}{crrrr}
\hline Variable & Lower bound & Upper bound & Initial value & Final value \\
\hline$\tau_{1}$ & -1.5 & -0.5 & -1 & -0.5 \\
$\tau_{2}$ & -1.5 & -0.5 & -1 & -0.5 \\
$\tau_{3}$ & -1.5 & -0.5 & -1 & -0.5 \\
$\tau_{4}$ & -1.5 & -0.5 & -1 & -0.83 \\
$\tau_{5}$ & -1.5 & -0.5 & -1 & -1.5 \\
$\tau_{6}$ & -1.5 & -0.5 & -1 & -0.83 \\
$\tau_{7}$ & -1.5 & -0.5 & -1 & -0.5 \\
$\tau_{8}$ & -1.5 & -0.5 & -1 & -0.5 \\
$\tau_{9}$ & -1.5 & -0.5 & -1 & -0.5 \\
\hline
\end{tabular}




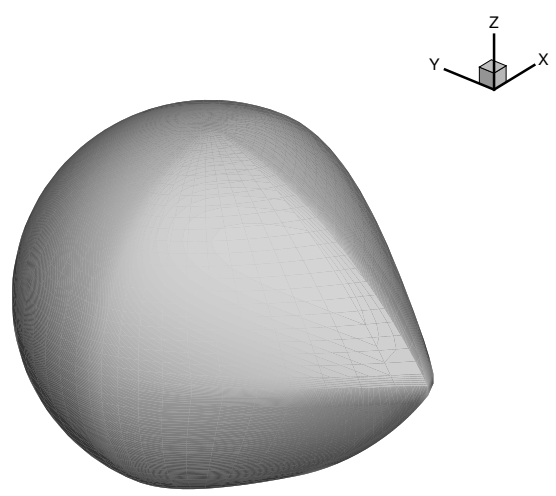

Fig. 12: The optimized shape of the sphere at $100 \mathrm{~Hz}$

\subsection{Scattering submarine model}

The numerical analysis of acoustic scattering characteristics of underwater submarines can effectively simulate the acoustic performance of submarines, and as such provide a necessary reference for the acoustic design of submarines. Herein, the Benchmark Target Strength Simulation Submarine (BeTSSi-Sub) shown in Fig. 13 is used for the numerical analysis. In this example, the acoustic scattering of an incident wave with a unit amplitude traveling along positive $y$ axis from the submarine is considered. The submarine model is given in Fig. 13a, and the control points of the NURBS surface representing the shape of the structure are presented in Fig. 13b. In this example, the sensitivity of the scattering acoustic pressure from the submarine model is computed, where the computing frequency is $100 \mathrm{~Hz}$ and the design variable is set as the $x$-coordinate of control point $\mathbf{P}_{4}$. The sensitivity contour plot is shown in Fig. 14 . The real and the imaginary parts of the sensitivity are shown in Figs. 14a and 14b, respectively. As expected, the sensitivity increases in the vicinity of the design control point.

After this initial verification, we address this submarine with the objective to min- 


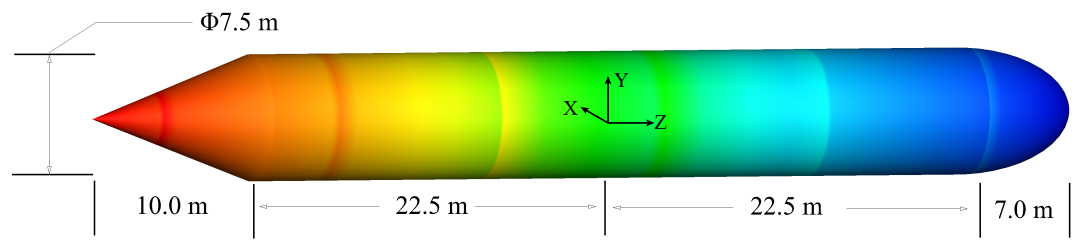

(a) The submarine model represented by NURBS: The origin of the coordinate system is located on the symmetrical axis.

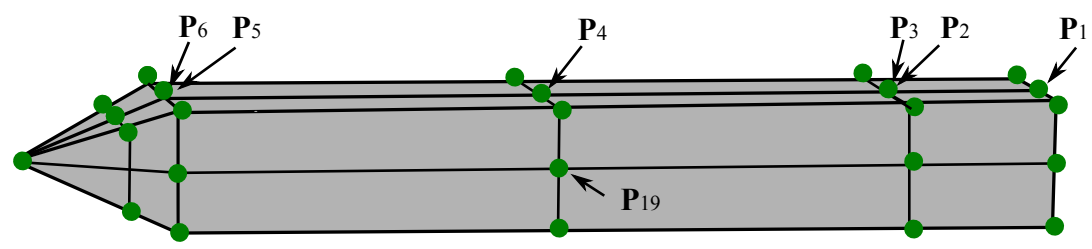

(b) The distribution of the control points constructuring the NURBS surface

Fig. 13: The submarine model represented by NURBS and the distribution of the control points: (a) The submarine model represented by NURBS consists of three parts. The left part is a conoid shell, the middle part is a cylindrical shell, and the right part is an elliptical shell; (b) The control point $\mathbf{P}_{4}$ is located on the positive $y$ axis. The origin of the coordinate system is located at the midpoint on the axis of the middle cylindrical shell.

imize the sound pressure at point $(10,10,0)$. The design variables are the $y$-direction coordinates of the control points $\left(\mathbf{P}_{1}-\mathbf{P}_{6}\right)$ on the top surface as depicted in Fig. 13b. It is noted that in the initial geometric configuration the positions of $\mathbf{P}_{2}$ and $\mathbf{P}_{3}$ coincide, and $\mathbf{P}_{5}$ and $\mathbf{P}_{6}$ also share the same initial coordinates. The volume constraint is $V \leqslant 2336.65$, and the side constraints of the design variables can be found in Table 2. Two different frequencies are used for the numerical solution. After the iterative process (Fig. 15 and Fig. 16), the optimized geometry and sound pressure distribution are obtained (Fig. 17). The final optimized design variables are listed in Table 2. Through the whole optimization procedure, the structural shape changes without mesh regeneration, which is a significant improvement in computational efficiency, robustness and ease of use of the method.

\subsection{Vase}

A vase model subject to a plane wave is shown in Fig. 18. The plane wave is traveling along the positive $y$-axis with unit amplitude. The design model is constructed 


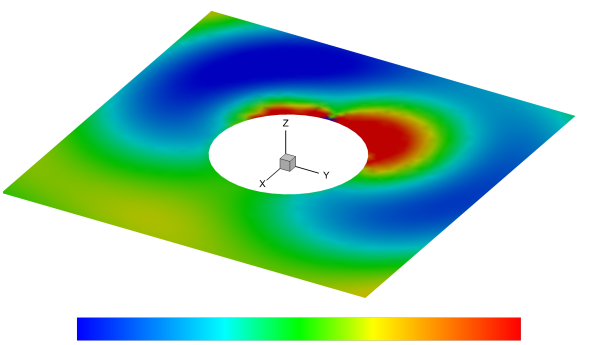

$\begin{array}{ccccccccccc}-0.1 & -0.08 & -0.06 & -0.04 & -0.02 & 0 & 0.02 & 0.04 & 0.06 & 0.08 & 0.1\end{array}$

(a) The real part of the sound pressure sensitivity

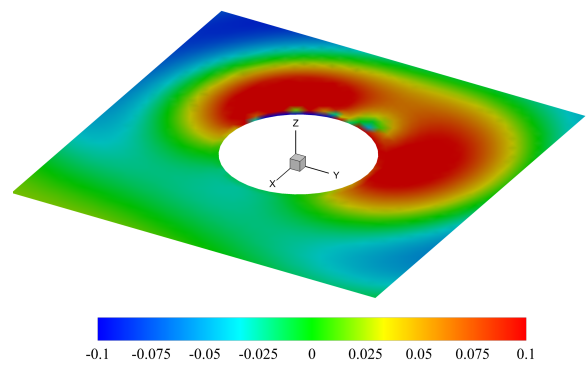

(b) The imaginary part of the sound pressure sensitivity

Fig. 14: The contour for the real part and the imaginary part of the sound pressure sensitivity on a square with a circular hole is plotted, respectively. This square is located on the xoy plane, and its size is $20 \times 20$ $\mathrm{m}^{2}$. The radius of the circular hole is $4 \mathrm{~m}$.

Table 2: Design variables in the submarine optimization procedure

\begin{tabular}{crrrrr}
\hline Design variable & Lower bound & Upper bound & Initial value & Final value $(50 \mathrm{~Hz})$ & Final value $(200 \mathrm{~Hz})$ \\
\hline$\tau_{1}$ & 1 & 6 & 3.75 & 1.85 & 1.00 \\
$\tau_{2}$ & 1 & 6 & 3.75 & 3.82 & 1.76 \\
$\tau_{3}$ & 1 & 6 & 3.75 & 1.41 & 3.70 \\
$\tau_{4}$ & 1 & 6 & 3.75 & 5.05 & 4.02 \\
$\tau_{5}$ & 1 & 6 & 3.75 & 4.81 & 3.00 \\
$\tau_{6}$ & 1 & 6 & 3.75 & 1.00 & 1.06 \\
\hline
\end{tabular}

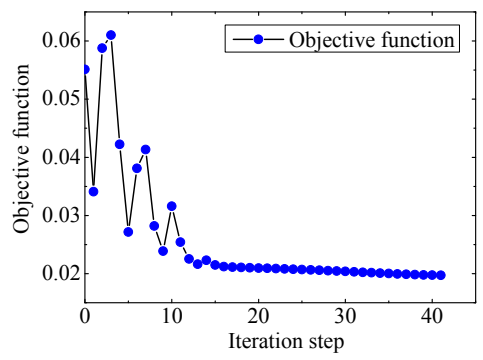

(a) $50 \mathrm{~Hz}$

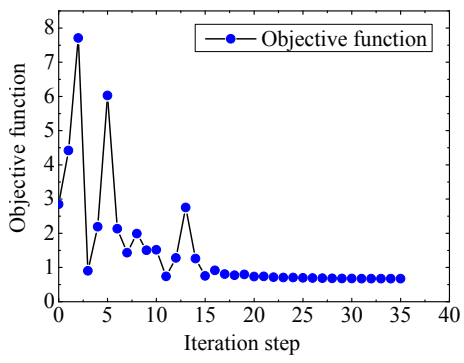

(b) $200 \mathrm{~Hz}$

Fig. 15: The convergence of the iterative process for the scattering submarine optimization problem. 


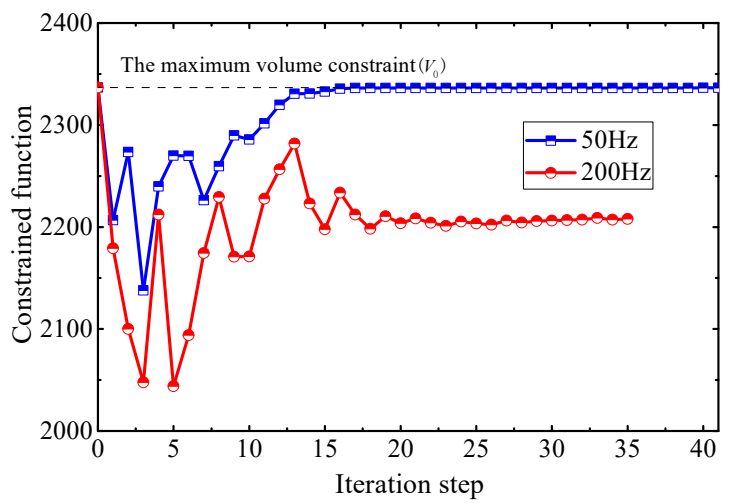

Fig. 16: Constrained function in terms of iteration steps for the scattering submarine optimization problem.

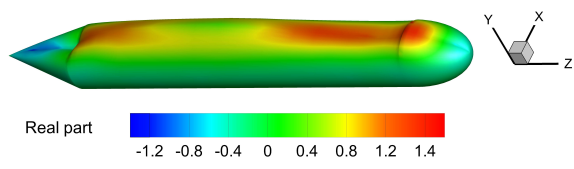

(a) Real part of sound pressure at $50 \mathrm{~Hz}$

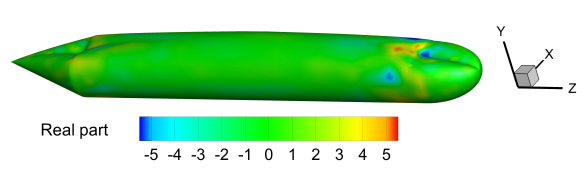

(c) Real part of sound pressure at $200 \mathrm{~Hz}$

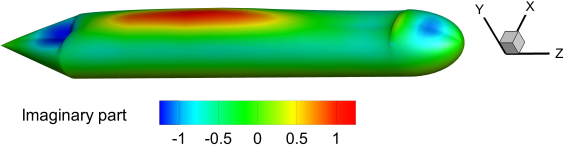

(b) Imaginary part of sound pressure at $50 \mathrm{~Hz}$

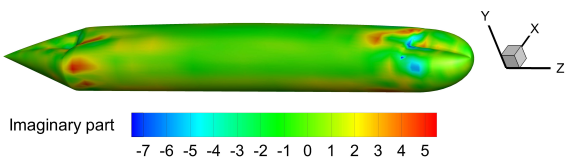

(d) Imaginary part of sound pressure at $200 \mathrm{~Hz}$

Fig. 17: The contour for the real part and the imaginary part of the sound pressure for the optimized geometry at two different frequencies.

using a quadratic NURBS surface with 126 control points. The control points selected for optimizing the shape are distributed on the design domain $S_{1}$ (Fig. 18). The $z$ direction coordinate value of the control points are chosen as the design variables. The initial value of the design variables on the $S_{1}$ surface are set as 4 . The lower bound and the upper bound are set to be 3.7 and 4.4, respectively (see Table 3). The constraint is that the structure volume $V$ should not exceed 32.57. The initial analysis mesh is refined from the design mesh. The objective is to minimize the sound pressure at point $(10,10,0)$. Fig. 19 and Fig. 20 illustrate the convergence of the iterative process, leading 


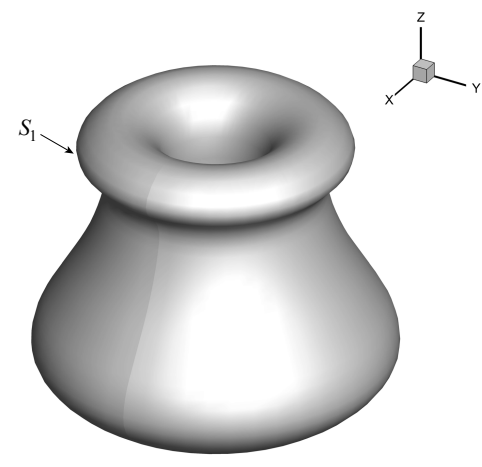

Fig. 18: Two knot vectors in two dimension are $\Xi=$ $[0,0,0,1 / 12,2 / 12,3 / 12,4 / 12,5 / 12,6 / 12,7 / 12,8 / 12,9 / 12,10 / 12,11 / 12,1,1,1], \quad$ and $\quad \Upsilon=$ $[0,0,0,0.25,0.25,0.5,0.5,0.75,0.75,1,1,1]$, respectively; The order is $p=2$ and $l=2$; The number of the control points is $126 ; S_{1}$ zone is used for the optimization analysis.

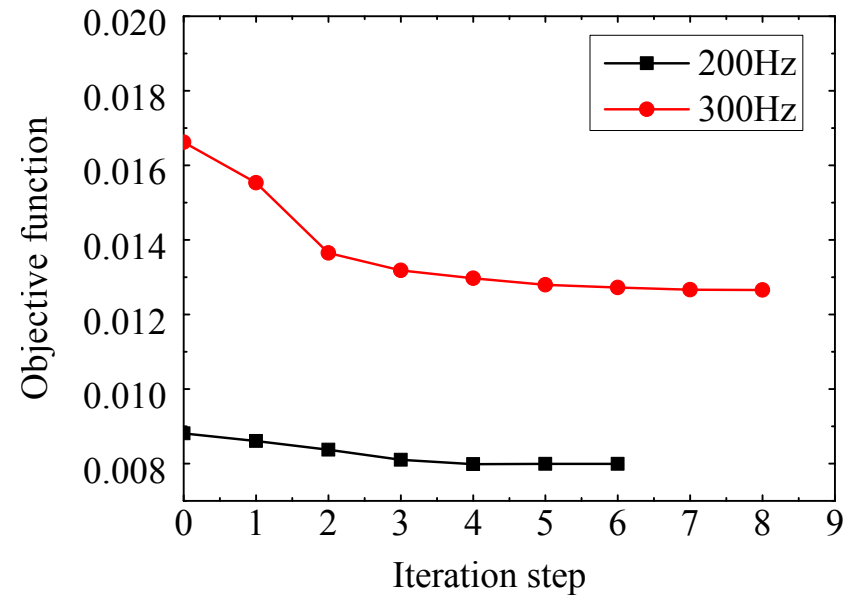

Fig. 19: The convergence of the iterative process for the vase optimization.

to the optimized geometry as shown in Fig. 21, compared to the initial geometry in Fig. 18. The final values of the design variables can be found in Table 3. The optimized shape is described by a NURBS geometric model which is returned to CAD designers for straightforward use without postprocessing. 


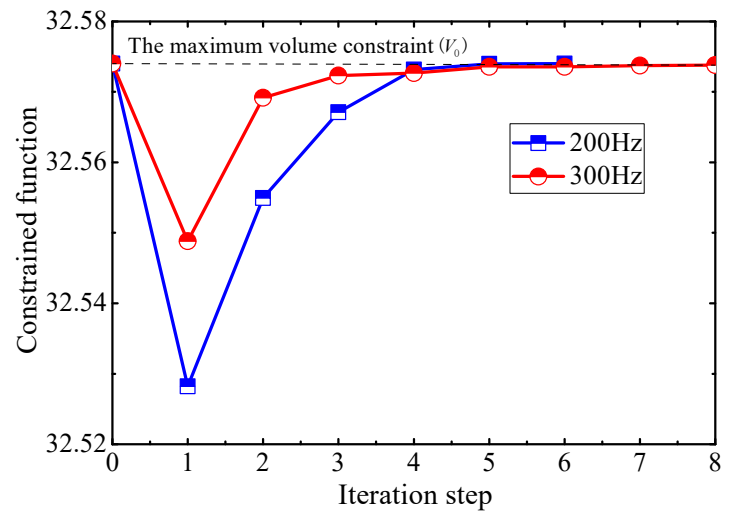

Fig. 20: Constrained function in terms of iteration steps for the scattering vase optimization problem.

Table 3: Design variables in the vase optimization procedure

\begin{tabular}{crrrrr}
\hline Design variable & Lower bound & Upper bound & Initial value & Final value $(200 \mathrm{~Hz})$ & Final value $(300 \mathrm{~Hz})$ \\
\hline$\tau_{1}$ & 3.7 & 4.4 & 4 & 3.80 & 3.83 \\
$\tau_{2}$ & 3.7 & 4.4 & 4 & 3.7 & 3.7 \\
$\tau_{3}$ & 3.7 & 4.4 & 4 & 4.4 & 3.7 \\
$\tau_{4}$ & 3.7 & 4.4 & 4 & 4.4 & 4.19 \\
$\tau_{5}$ & 3.7 & 4.4 & 4 & 3.7 & 4.19 \\
$\tau_{6}$ & 3.7 & 4.4 & 4 & 4.09 & 4.05 \\
$\tau_{7}$ & 3.7 & 4.4 & 4 & 3.7 & 4.4 \\
\hline
\end{tabular}

\section{Conclusion}

This work extended the isogeometric boundary element method (IGABEM) to three dimensional acoustic shape optimization problems. The main strength of the algorithm lies in the ability of IGABEM in simulating infinite domain problems and seamlessly integrating CAD and numerical analysis. No mesh is generated at any time and geometrical exactness is preserved. The control points are chosen as design variables naturally, and the optimized solution can return CAD models automatically without geometry postprocessing. To remove the non-uniqueness in exterior domain problems, the Burton-Miller formulation is adopted but raises the difficulties of hyper- 


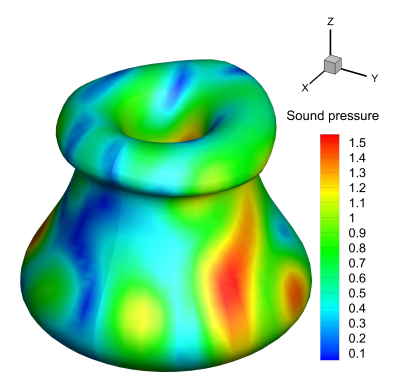

(a) $f=200 \mathrm{~Hz}$

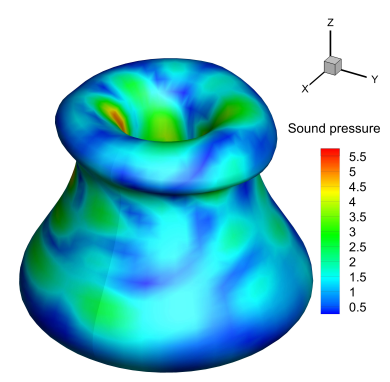

(b) $f=300 \mathrm{~Hz}$

Fig. 21: The optimized shape of the vase model with two different computing frequencies.

singular integrals. The singular integrals in Burton-Miller formulas are evaluated explicitly by using the method presented by [25]. The shape sensitivity formulation for IGABEM is derived through the implicit differentiation method, and the design variables are the coordinates of control points. The proposed shape optimization methods was validated through several numerical examples. However, this work is not without limitations. Due to the full populated matrices in BEM formulation and the recursion formula of NURBS basis functions, the present work has high computational cost, which hinders its application in large scale problems. In the future, the assembly of the matrices can be accelerated using Fast Multipole Method [50], Adaptive Crossing Approximation [4], and pre-Corrected Fast Fourier Transformation [48]. Bézier extraction will be employed for fast evaluation of basis function. Moreover, the surrogate model and model order reduction techniques can be employed to improve the efficiency of shape optimization. [31,30]. The application of shape optimization in electromagnetics is also a direction to pursue and a natural extension of this work.

\section{Acknowledgements}

Financial supports from the National Natural Science Foundation of China (NSFC) under Grant No. 11702238, the Henan Provincial Department of Science and Tech- 
nology Research under Grant no. 172102210453, Key Scientific Research Project of Henan University under Grant no. 17A560009, and Nanhu Scholars Program for Young Scholars of XYNU are acknowledged. Stéphane Bordas thanks the financial support of the European Research Council Starting Independent Research Grant (ERC Stg grant agreement No. 279578) entitled Towards real time multiscale simulation of cutting in non-linear materials with applications to surgical simulation and computer guided surgery" and the support of the Fonds National de la Recherche Luxembourg INTER/FNRS/15/11019432/EnLightenIt/Bordas. Haibo Chen appreciates the support of the National Natural Science Foundation of China (NSFC) under Grant No. 11772322.

\section{Appendix A: Evaluation of the coefficient functions}

First, $x_{i}-s_{i}$ can be expanded as follows

$$
x_{i}-s_{i}=\rho A_{i}(\theta)+\rho^{2} B_{i}(\theta)+O\left(\rho^{3}\right),
$$

where

$$
A_{i}(\theta)=\left.\cos (\theta) \frac{\partial s_{i}}{\partial \xi}\right|_{\substack{\xi=\hat{\xi} \\ \eta=\hat{\eta}}}+\left.\sin (\theta) \frac{\partial s_{i}}{\partial \eta}\right|_{\substack{\xi=\hat{\xi} \\ \eta=\hat{\eta}}}
$$

and

$$
B_{i}(\theta)=\left.\frac{\cos ^{2}(\theta)}{2} \frac{\partial^{2} s_{i}}{\partial \xi^{2}}\right|_{\substack{\xi=\hat{\xi} \\ \eta=\hat{\eta}}}+\left.\cos (\theta) \sin (\theta) \frac{\partial^{2} s_{i}}{\partial \xi \partial \eta}\right|_{\substack{\xi=\hat{\xi} \\ \eta=\hat{\eta}}}+\left.\frac{\sin ^{2}(\theta)}{2} \frac{\partial^{2} s_{i}}{\partial \eta^{2}}\right|_{\substack{\xi=\hat{\xi} \\ \eta=\hat{\eta}}}
$$

Similarly, $r=|x-s|$ can be expanded as follows

$$
r^{n}=\rho^{n} A^{n}(\theta)\left[1+n \rho \frac{C(\theta)}{A^{2}(\theta)}\right]+O\left(\rho^{n+2}\right)
$$


where

$$
\begin{aligned}
& A(\theta)=\left[A_{1}^{2}(\theta)+A_{2}^{2}(\theta)+A_{3}^{2}(\theta)\right]^{1 / 2}, \\
& B(\theta)=\left[B_{1}^{2}(\theta)+B_{2}^{2}(\theta)+B_{3}^{2}(\theta)\right]^{1 / 2}, \\
& C(\theta)=A_{1}(\theta) B_{1}(\theta)+A_{2}(\theta) B_{2}(\theta)+A_{3}(\theta) B_{3}(\theta) .
\end{aligned}
$$

When $n=-3$, we can the expansion formulation of $r^{-3}$, as follows

$$
\frac{1}{r^{3}}=\frac{S_{3}(\theta)}{\rho^{3}}+\frac{S_{2}(\theta)}{\rho^{2}}+O\left(\frac{1}{\rho}\right)
$$

where

$$
\begin{aligned}
& S_{3}(\theta)=A^{-3}(\theta), \\
& S_{2}(\theta)=-\frac{3 C(\theta)}{A^{5}(\theta)} .
\end{aligned}
$$

NURBS basis function is expanded as

$$
\begin{aligned}
R_{A}(\xi, \eta) & =R_{A}(\hat{\xi}, \hat{\eta})+\rho\left[\left.\cos (\theta) \frac{\partial R_{A}}{\partial \xi}\right|_{\substack{\xi=\hat{\xi} \\
\eta=\hat{\eta}}}+\left.\sin (\theta) \frac{\partial R_{A}}{\partial \eta}\right|_{\substack{\xi=\hat{\xi} \\
\eta=\hat{\eta}}}\right]+O\left(\rho^{2}\right) \\
& =R_{A}^{0}+\rho R_{a}^{1}+O\left(\rho^{2}\right)
\end{aligned}
$$

And then, $n_{i}(x) n_{i}(y) J$ of the function $f(\theta, \rho)$ can be expressed in the parametric space as follows

$$
n_{i}(\mathbf{s}) n_{i}(\mathbf{x}) J=n_{i}(\hat{\xi}, \hat{\eta}) n_{i}(\xi, \eta) J(\xi, \eta)=n_{i}(\hat{\xi}, \hat{\eta}) J_{i}(\xi, \eta)
$$


By using the Taylor's expansion procedure, we can obtain the following formulation

$$
\begin{aligned}
n_{i}(\hat{\xi}, \hat{\eta}) J_{i}(\xi, \eta) & =n_{i}(\hat{\xi}, \hat{\eta}) J_{i}(\hat{\xi}, \hat{\eta})+\rho n_{i}(\hat{\xi}, \hat{\eta})\left[\left.\cos (\theta) \frac{\partial J_{i}}{\partial \xi}\right|_{\substack{\xi=\hat{\xi} \\
\eta=\hat{\eta}}}+\left.\sin (\theta) \frac{\partial J_{i}}{\partial \eta}\right|_{\substack{\xi=\hat{\xi} \\
\eta=\hat{\eta}}}\right]+O\left(\rho^{2}\right) \\
& =J(\hat{\xi}, \hat{\eta})+\rho n_{i}(\hat{\xi}, \hat{\eta}) J_{i 1}(\hat{\xi}, \hat{\eta})+O\left(\rho^{2}\right) \\
& =J_{0}+\rho J_{01}+O\left(\rho^{2}\right)
\end{aligned}
$$

By substituting Eqs. (73), (76), and (78) into Eq. (35), we can obtain the solution of the functions $f_{1}(\theta)$ and $f_{2}(\theta)$, as follows

$$
\begin{aligned}
& f_{1}(\theta)=\frac{S_{2} R_{A}^{0} J_{0}+S_{3}\left(R_{A}^{1} J_{0}+R_{A}^{0} J_{01}\right)}{4 \pi}, \\
& f_{2}(\theta)=\frac{S_{3} R_{A}^{0} J_{0}}{4 \pi} .
\end{aligned}
$$

Using Eq. (69) and setting $n=1$ and $r=\varepsilon$, we can obtain the expansion expression of $\varepsilon$, as follows

$$
\varepsilon=\rho A(\theta)+\rho^{2} \frac{C(\theta)}{A(\theta)}+O\left(\rho^{3}\right)
$$

And then, by substituting Eq. (81) into Eq. (37), we can obtain the solution of $\beta(\theta)$ and $\gamma(\theta)$, as follows

$$
\begin{aligned}
& \beta(\theta)=A^{-1}(\theta) \\
& \gamma(\theta)=-\frac{C(\theta)}{A^{4}(\theta)} .
\end{aligned}
$$

At present, we have obtained solution of all unknown coefficients $f_{1}(\theta), f_{2}(\theta), \beta(\theta)$, and $\gamma(\theta)$ in Eq. (41). 


\section{Appendix B: Evaluation of singular sensitivity boundary integral}

Actually, we can find that the singularity order of sensitivities of the kernel functions are same as that of kernel functions by comparing Eqs. (17-18) with Eqs. (44-47). So, the functions for $G, F, K, \dot{G}, \dot{F}$, and $\dot{K}$ are weakly singular, and the functions for $H$ and $\dot{H}$ are hypersingular. For the weakly singular boundary integrals, a polar integration scheme can be applied to eliminate the singularity. Please see section (3.2.1). Herein, the procedure for evaluation of the hypersingular boundary integral with $\dot{H}$ kernel function is presented, similarly to that of the boundary integral with $H$ kernel function.

By using polar coordinate transformation, the hypersingular boundary integral with $\dot{H}$ kernel function in Eq. (43) can be expressed as

$$
\int_{\xi_{e}}^{\xi_{e+1}} \int_{\eta_{e}}^{\eta_{e+1}} \dot{H} R_{A} J \mathrm{~d} \xi \mathrm{d} \eta=\int_{0}^{2 \pi} \int_{0}^{\rho(\theta)} \dot{H} R_{A} J \rho \mathrm{d} \rho \mathrm{d} \theta
$$

By observing the expression of $\dot{H}$ kernel function, we can find that the highest singular term is

$$
-\frac{3 \mathrm{e}^{\mathrm{i} k r} n_{i}(\mathbf{s}) n_{i}(\mathbf{x}) \dot{r}}{4 \pi r^{4}}
$$

when $\rho$ is very small, we can obtain the following formulas

$$
\frac{3 n_{i}(\mathbf{s}) n_{i}(\mathbf{x}) \dot{r} R_{A} J \rho}{4 \pi r^{4}}=\frac{u_{2}(\theta)}{\rho^{2}}+\frac{u_{1}(\theta)}{\rho}+O\left(\rho^{0}\right) .
$$

Similarly as Eq. (36), we can obtain the following formulas

$$
\begin{array}{r}
\int_{\xi_{e}}^{\xi_{e+1}} \int_{\eta_{e}}^{\eta_{e+1}} \dot{H} R_{A} J \mathrm{~d} \xi \mathrm{d} \eta=\int_{0}^{2 \pi} \int_{0}^{\rho_{2}(\theta)}\left[\dot{H} R_{A} J \rho+\left(\frac{u_{2}(\theta)}{\rho^{2}}+\frac{u_{1}(\theta)}{\rho}\right)\right] \mathrm{d} \rho \mathrm{d} \theta \\
-\int_{0}^{2 \pi} u_{1}(\theta) \ln \left|\frac{\rho_{2}(\theta)}{\beta(\theta)}\right| \mathrm{d} \theta-\int_{0}^{2 \pi} u_{2}(\theta)\left[\frac{\gamma(\theta)}{\beta^{2}(\theta)}-\frac{1}{\rho_{2}(\theta)}\right] \mathrm{d} \theta
\end{array}
$$


where all the integrals are nonsingular, which means the use of Gauss-Legendre integral method is allowed. The expression of $\beta(\theta)$ and $\gamma(\theta)$ are presented in Eqs. (82) and (83), respectively. But we still need to obtain the solution of $u_{1}(\theta)$ and $u_{2}(\theta)$. According to Eq. (69), when $n=-4$, we can obtain the following formulas

$$
\frac{1}{r^{4}}=\frac{S_{4}(\theta)}{\rho^{4}}+\frac{\tilde{S}_{3}(\theta)}{\rho^{3}}+O\left(\frac{1}{\rho^{2}}\right)
$$

where

$$
\begin{aligned}
& S_{4}(\theta)=A^{-4}(\theta), \\
& \tilde{S}_{3}(\theta)=-\frac{4 C(\theta)}{A^{6}(\theta)} .
\end{aligned}
$$

$\dot{r}$ can also be expanded as

$$
\begin{aligned}
\dot{r} & =\frac{\left(x_{i}-s_{i}\right)\left(\dot{x}_{i}-\dot{s}_{i}\right)}{r}=\frac{\left(\rho A_{i}(\theta)+\rho^{2} B_{i}(\theta)+O\left(\rho^{3}\right)\right)\left(\rho \dot{A}_{i}(\theta)+\rho^{2} \dot{B}_{i}(\theta)+O\left(\rho^{3}\right)\right)}{\rho A(\theta)\left(1+\rho \frac{C(\theta)}{A^{2}(\theta)}\right)+O\left(\rho^{3}\right)} \\
& =\rho \frac{A_{i}(\theta) \dot{A}_{i}(\theta)}{A(\theta)}+\rho^{2}\left(\frac{A_{i}(\theta) \dot{B}_{i}(\theta)+\dot{A}_{i}(\theta) B_{i}(\theta)}{A(\theta)}-\frac{C(\theta)\left(A_{i}(\theta) \dot{A}_{i}(\theta)\right)}{A^{4}(\theta)}\right)+O\left(\rho^{3}\right) \\
& =\rho\left(S_{0}+\rho S_{1}\right)+O\left(\rho^{3}\right)
\end{aligned}
$$

By substituting Eqs. (91), (88), (78), and (76) into Eq. (86), we can obtain the following formulas

$$
\begin{aligned}
\frac{3 n_{i}(\mathbf{s}) n_{i}(\mathbf{x}) \dot{r} R_{A} J \rho}{4 \pi r^{4}}= & \frac{3}{4 \pi} \times\left[\frac{S_{4}}{\rho^{4}}+\frac{\tilde{S}_{3}}{\rho^{3}}+O\left(\frac{1}{\rho^{2}}\right)\right] \times\left[\rho\left(S_{0}+\rho S_{1}\right)+O\left(\rho^{3}\right)\right] \times \\
& {\left[R_{A}^{0}+\rho R_{A}^{1}+O\left(\rho^{2}\right)\right] \times\left[J_{0}+\rho J_{01}+O\left(\rho^{2}\right)\right] \times \rho } \\
& =\frac{3 S_{4} S_{0} R_{a}^{0} J_{0}}{4 \pi \rho^{2}}+\frac{3\left[\tilde{S}_{3} S_{0} R_{A}^{0} J_{0}+S_{4}\left(R_{A}^{1} J_{0} S_{0}+J_{01} S_{0} R_{A}^{0}+S_{1} R_{A}^{0} J_{0}\right)\right]}{4 \pi \rho}+O\left(\rho^{0}\right) .
\end{aligned}
$$


By substituting Eq. (92) into Eq. (86), we can obtain the expression of $u_{1}(\theta)$ and $u_{2}(\theta)$, as follows

$$
\begin{aligned}
& u_{1}(\theta)=\frac{3}{4 \pi}\left[\tilde{S}_{3} S_{0} R_{A}^{0} J_{0}+S_{4}\left(R_{A}^{1} J_{0} S_{0}+J_{01} S_{0} R_{A}^{0}+S_{1} R_{A}^{0} J_{0}\right)\right], \\
& u_{2}(\theta)=\frac{3}{4 \pi} S_{4} S_{0} R_{A}^{0} J_{0} .
\end{aligned}
$$

Finally, we can obtain the solution of the hypersingular sensitivity boundary integral with $\dot{H}$ kernel function by using Eq. (87).

\section{References}

[1] Y. Bazilevs, V.M. Calo, J.A. Cottrell, J.A. Evans, T.J.R. Hughes, S. Lipton, M. A. Scott, and T. W. Sederberg. Isogeometric analysis using T-splines. Computers and Mathematics with Applications, 199(5-8):229-263, 2010.

[2] Y. Bazilevs, V.M. Calo, T.J.R. Hughes, and Y. Zhang. Isogeometric fluid-structure interaction: Theory, algorithms, and computations. Computational Mechanics, 43:3-37, 2008.

[3] Y. Bazilevs, C. Michler, V.M. Calo, and T.J.R. Hughes. Isogeometric variational multiscale modeling of wall-bounded turbulent flows with weakly enforced boundary conditions on unstretched meshes. Computer Methods in Applied Mechanics and Engineering, 199(13-16):780-790, 2010.

[4] M. Bebendorf. Approximation of boundary element matrices. Numerische Mathematik, 86(4):565-589, 2000.

[5] G. Beer, V. Mallardo, E. Ruocco, B. Marussig, J. Zechner, C. Dünser, and T. Fries. Isogeometric boundary element analysis with elasto-plastic inclusions. part 2: 3-D problems. Computer Methods in Applied Mechanics and Engineering, 315:418-433, 2017. 
[6] G. Beer, B. Marussig, J. Zechner, C. Dünser, and T. Fries. Isogeometric boundary element analysis with elasto-plastic inclusions. part 1: plane problems. Computer Methods in Applied Mechanics and Engineering, 308:552-570, 2016.

[7] D.J. Benson, Y. Bazilevs, M.C. Hsu, and T.J.R. Hughes. Isogeometric shell analysis: The Reissner-Mindlin shell. Computer Methods in Applied Mechanics and Engineering, 199(5-8):276-289, 2010.

[8] H. Bériot, E. Perrey-Debain, M.B. Tahar, and C. Vayssade. Plane wave basis in Galerkin BEM for bidimensional wave scattering. Engineering Analysis with Boundary Elements, 34(2):130-143, 2010.

[9] G. Beylkin, R. Coifman, and V. Rokhlin. Fast wavelet transforms and numerical algorithms I. Communications on Pure and Applied Mathematics, 44(2):141$183,1991$.

[10] S.P.A. Bordas, T. Rabczuk, J.J. Ródenas, P. Kerfriden, M. Moumnassi, and S. Belouettar. Alleviating the mesh burden in computational solid mechanics. Proceedings of ECT2010, 2010.

[11] L.L. Chen, H.B. Chen, C.J. Zheng, and S. Marburg. Structural-acoustic sensitivity analysis of radiated sound power using a finite element/ discontinuous fast multipole boundary element scheme. International journal for numerical methods in fluids, 82:858-878, 2016.

[12] L.L. Chen, C. Liu, W.C. Zhao, and Liu L.C. An isogeometric approach of two dimensional acoustic design sensitivity analysis and topology optimization analysis for absorbing material distribution. Computer Methods in Applied Mechanics and Engineering, 336:507-532, 2018.

[13] L.L. Chen, S. Marburg, H.B. Chen, H. Zhang, and H. Gao. An adjoint operator ap- 
proach for sensitivity analysis of radiated sound power in fully coupled structuralacoustic systems. Journal of Computational Acoustics, 25(01):1750003, 2017.

[14] L.L. Chen, C.J. Zheng, and H.B. Chen. FEM/wideband FMBEM coupling for structural-acoustic design sensitivity analysis. Computer Methods in Applied Mechanics and Engineering, 276:1-19, 2014.

[15] A.N. Christiansen, J.A. Bærentzen, M. Nobel-Jørgensen, N. Aage, and O. Sigmund. Combined shape and topology optimization of $3 \mathrm{~d}$ structures. Computers \& Graphics, 46:25-35, 2015.

[16] A.N. Christiansen, M. Nobel-Jørgensen, N. Aage, O. Sigmund, and J.A. Bærentzen. Topology optimization using an explicit interface representation. Structural and Multidisciplinary Optimization, 49(3):387-399, 2014.

[17] F. Cirak, M.J. Scott, E.K. Antonsson, M. Ortiz, and P. Schröder. Integrated modeling, finite-element analysis, and engineering design for thin-shell structures using subdivision. Computer-Aided Design, 34(2):137-148, 2002.

[18] J.A. Cottrell, A. Reali, Y. Bazilevs, and T.J.R. Hughes. Isogeometric analysis of structural vibrations. Computer Methods in Applied Mechanics and Engineering, 195(41-43):5257-5296, 2006.

[19] L. De Lorenzis, I. Temizer, P. Wriggers, and G. Zavarise. A large deformation frictional contact formulation using NURBS-based isogeometric analysis. International Journal for Numerical Methods in Engineering, 2011.

[20] M. Feischl, G. Gantner, A. Haberl, and D. Praetorius. Optimal convergence for adaptive IGA boundary element methods for weakly-singular integral equations. Numerische Mathematik, 136(1):147-182, 2017.

[21] M. Feischl, G. Gantner, and D. Praetorius. Reliable and efficient a posteriori error estimation for adaptive IGA boundary element methods for weakly-singular 
integral equations. Computer Methods in Applied Mechanics and Engineering, 290:362-386, 2015.

[22] M. Fischer and L. Gaul. Fast BEM-FEM mortar coupling for acousticstructure interaction. International Journal for Numerical Methods in Engineering, 62(12):1677-1690, 2005.

[23] A.I. Ginnis, K.V. Kostas, C.G. Politis, P.D. Kaklis, K.A. Belibassakis, Th.P. Gerostathis, M.A. Scott, and T.J.R. Hughes. Isogeometric boundary-element analysis for the wave-resistance problem using T-splines. Computer Methods in Applied Mechanics and Engineering, 279:425-439, 2014.

[24] L. Greengard and V. Rokhlin. A fast algorithm for particle simulations. Journal of Computational Physics, 73(2):325-348, 1987.

[25] M. Guiggiani and P. Casalini. Direct computation of Cauchy principal value integrals in advanced boundary elements. International Journal for Numerical Methods in Engineering, 24(9):1711-1720, 1987.

[26] T.J.R. Hughes, J.A. Cottrell, and Y. Bazilevs. Isogeometric analysis: CAD, finite elements, NURBS, exact geometry and mesh refinement. Computer Methods in Applied Mechanics and Engineering, 194(39-41):4135-4195, 2005.

[27] T. Khajah, X. Antoine, and S. Bordas. Isogeometric finite element analysis of time-harmonic exterior acoustic scattering problems. arXiv preprint arXiv:1610.01694, 2016.

[28] K.V. Kostas, A.I. Ginnis, C.G. Politis, and P.D. Kaklis. Ship-hull shape optimization with a T-spline based BEM-isogeometric solver. Computer Methods in Applied Mechanics and Engineering, 284:611-622, 2015.

[29] K. Li and X. Qian. Isogeometric analysis and shape optimization via boundary integral. Computer-Aided Design, 43(11):1427-1437, 2011. 
[30] S. Li, J. Trevelyan, Z. Wu, H. Lian, D. Wang, and W. Zhang. An adaptive SVDKrylov reduced order model for surrogate based structural shape optimization through isogeometric boundary element method. Computer Methods in Applied Mechanics and Engineering, 349:312-338, 2019.

[31] S. Li, J. Trevelyan, W. Zhang, and D. Wang. Accelerating isogeometric boundary element analysis for 3-dimensional elastostatics problems through black-box fast multipole method with proper generalized decomposition. International Journal for Numerical Methods in Engineering, 114(9):975-998, 2018.

[32] H. Lian, A.N. Christiansen, D.A. Tortorelli, O. Sigmund, and N. Aage. Combined shape and topology optimization for minimization of maximal von Mises stress. Structural and Multidisciplinary Optimization, 55(5):1541-1557, 2017.

[33] H. Lian, P. Kerfriden, and S.P.A. Bordas. Implementation of regularized isogeometric boundary element methods for gradient-based shape optimization in two-dimensional linear elasticity. International Journal for Numerical Methods in Engineering, 106(12):972-1017, 2016.

[34] H. Lian, P. Kerfriden, and S.P.A. Bordas. Shape optimization directly from CAD: An isogeometric boundary element approach using T-splines. Computer Methods in Applied Mechanics and Engineering, 317:1-41, 2017.

[35] C. Liu, L. Chen, W. Zhao, and H. Chen. Shape optimization of sound barrier using an isogeometric fast multipole boundary element method in two dimensions. Engineering Analysis with Boundary Elements, 85:142157, 2017.

[36] Z. Liu, M. Majeed, F. Cirak, and R.N. Simpson. Isogeometric fem-bem coupled structural-acoustic analysis of shells using subdivision surfaces. International Journal for Numerical Methods in Engineering, 113(9):1507-1530, 2018. 
[37] C. Marot, J. Pellerin, and J-F Remacle. One machine, one minute, three billion tetrahedra. arXiv preprint arXiv:1805.08831, 2018.

[38] S. Natarajan, E.T. Ooi, A. Saputra, and C Song. A scaled boundary finite element formulation over arbitrary faceted star convex polyhedra. Engineering Analysis with Boundary Elements, 80:218-229, 2017.

[39] B.H. Nguyen, H.D. Tran, C. Anitescu, X. Zhuang, and T. Rabczuk. An isogeometric symmetric galerkin boundary element method for two-dimensional crack problems. Computer Methods in Applied Mechanics and Engineering, 306:252$275,2016$.

[40] V.P. Nguyen, C. Anitescu, S.P.A Bordas, and T. Rabczuk. Isogeometric analysis: an overview and computer implementation aspects. Mathematics and Computers in Simulation, 117:89-116, 2015.

[41] V.P. Nguyen, P. Kerfriden, M. Brino, S.P. A. Bordas, and E. Bonisoli. Nitsche's method for two and three dimensional NURBS patch coupling. Computational Mechanics, 53(6):1163-1182, 2014.

[42] N. Nguyen-Thanh, J. Kiendl, H. Nguyen-Xuan, R. Wüchner, K.U. Bletzinger, Y. Bazilevs, and T. Rabczuk. Rotation free isogeometric thin shell analysis using PHT-splines. Computer Methods in Applied Mechanics and Engineering, 200(47):3410-3424, 2011.

[43] M.J. Peake, J. Trevelyan, and G. Coates. Novel basis functions for the partition of unity boundary element method for helmholtz problems. International Journal for Numerical Methods in Engineering, 93(9):905-918, 2013.

[44] M.J. Peake, J. Trevelyan, and G. Coates. Extended isogeometric boundary element method (XIBEM) for three-dimensional medium-wave acoustic scattering 
problems. Computer Methods in Applied Mechanics and Engineering, 284:762$780,2015$.

[45] X. Peng, E. Atroshchenko, P. Kerfriden, and S.P.A. Bordas. Isogeometric boundary element methods for three dimensional static fracture and fatigue crack growth. Computer Methods in Applied Mechanics and Engineering, 316:151$185,2017$.

[46] X. Peng, E. Atroshchenko, P. Kerfriden, and S.P.A. Bordas. Linear elastic fracture simulation directly from CAD: 2D NURBS-based implementation and role of tip enrichment. International Journal of Fracture, 204(1):55-78, 2017.

[47] E. Perrey-Debain, J. Trevelyan, and P. Bettess. Plane wave interpolation in direct collocation boundary element method for radiation and wave scattering: numerical aspects and applications. Journal of Sound and Vibration, 261(5):839-858, 2003.

[48] J.R. Phillips and J.K. White. A precorrected-FFT method for electrostatic analysis of complicated 3-D structures. IEEE Transactions on Computer-Aided Design of Integrated Circuits and Systems, 16(10):1059-1072, 1997.

[49] C. Politis, A.I. Ginnis, P.D. Kaklis, K. Belibassakis, and C. Feurer. An isogeometric BEM for exterior potential-flow problems in the plane. In Proceedings of SIAM/ACM joint conference on geometric and physical modeling, 2009.

[50] V. Rokhlin. Rapid solution of integral equations of classical potential theory. Journal of computational physics, 60(2):187-207, 1985.

[51] M.A. Scott, M.J. Borden, C.V. Verhoosel, T.W. Sederberg, and T.J.R. Hughes. Isogeometric finite element data structures based on Bézier extraction of $\mathrm{T}$ splines. International Journal for Numerical Methods in Engineering, 88(2):126156, 2011. 
[52] M.A. Scott, R.N. Simpson, J.A. Evans, S. Lipton, S.P.A Bordas, T.J.R. Hughes, and T.W. Sederberg. Isogeometric boundary element analysis using unstructured T-splines. Computer Methods in Applied Mechanics and Engineering, 254:197$221,2013$.

[53] R.N. Simpson, S.P.A. Bordas, H. Lian, and J. Trevelyan. An isogeometric boundary element method for elastostatic analysis: 2D implementation aspects. Computers \& Structures, 118:2-12, 2013.

[54] R.N. Simpson, S.P.A. Bordas, J. Trevelyan, and T. Rabczuk. A two-dimensional isogeometric boundary element method for elastostatic analysis. Computer Methods in Applied Mechanics and Engineering, 209-212:87-100, 2012.

[55] R.N. Simpson and Z. Liu. Acceleration of isogeometric boundary element analysis through a black-box fast multipole method. Engineering Analysis with Boundary Elements, 66:168-182, 2016.

[56] R.N. Simpson, Z. Liu, R. Vazquez, and J.A. Evans. An isogeometric boundary element method for electromagnetic scattering with compatible B-spline discretizations. Journal of Computational Physics, 362:264-289, 2018.

[57] R.N. Simpson, M.A. Scott, M. Taus, D.C. Thomas, and H. Lian. Acoustic isogeometric boundary element analysis. Computer Methods in Applied Mechanics and Engineering, 269:265-290, 2014.

[58] K. Sören, N.C. Hagelstein, O. Zaleski, and O. Von Estorff. Evaluation of hypersingular and nearly singular integrals in the isogeometric boundary element method for acoustics. Computer Methods in Applied Mechanics and Engineering, 325:488-504, 2017.

[59] S. Sun, T. Yu, T.T. Nguyen, E. Atroshchenko, and T.Q. Bui. Structural shape op- 
timization by IGABEM and particle swarm optimization algorithm. Engineering Analysis with Boundary Elements, 88:26-40, 2018.

[60] K. Svanberg. The method of moving asymptotes-a new method for structural optimization. International Journal for Numerical Methods in Engineering, 24:359373, 1987.

[61] M.Y. Wang, X. Wang, and D. Guo. A level set method for structural topology optimization. Computer Methods in Applied Mechanics and Engineering, 192(1):227-246, 2003.

[62] G. Xu, B. Mourrain, R. Duvigneau, and A. Galligo. Constructing analysissuitable parameterization of computational domain from CAD boundary by variational harmonic method. Journal of Computational Physics, 252:275-289, 2013.

[63] G. Xu, B. Mourrain, R. Duvigneau, and A. Galligo. Optimal analysis-aware parameterization of computational domain in 3D isogeometric analysis. ComputerAided Design, 45(4):812-821, 2013.

[64] W. Zhao, L. Chen, H. Chen, and S. Marburg. Topology optimization of exterior acoustic-structure interaction systems using the coupled FEM-BEM method. International Journal for Numerical Methods in Engineering, 82(12):858-878, 2019.

[65] C. Zheng, C. Bi, C. Zhang, Y. Zhang, and H. Chen. Fictitious eigenfrequencies in the BEM for interior acoustic problems. Engineering Analysis With Boundary Elements, 104:170-182, 2019.

[66] M. Zhou, H. Lian, O. Sigmund, and N. Aage. Shape morphing and topology optimization of fluid channels by explicit boundary tracking. International Journal for Numerical Methods in Fluids, 88(6):296-313, 2018. 